ARTICLE

\title{
Increasing ambient temperature progressively disassembles Arabidopsis phytochrome B from individual photobodies with distinct thermostabilities
}

\author{
Joseph Hahm ${ }^{1,2}$, Keunhwa Kim ${ }^{1,2}$, Yongjian Qiu (1D ${ }^{1} \&$ Meng Chen (1D) ${ }^{1 凶}$
}

Warm temperature is postulated to induce plant thermomorphogenesis through a signaling mechanism similar to shade, as both destabilize the active form of the photoreceptor and thermosensor phytochrome B (phyB). At the cellular level, shade antagonizes phyB signaling by triggering phy $B$ disassembly from photobodies. Here we report temperature-dependent photobody localization of fluorescent protein-tagged phyB (phyB-FP) in the epidermal cells of Arabidopsis hypocotyl and cotyledon. Our results demonstrate that warm temperature elicits different photobody dynamics than those by shade. Increases in temperature from $12{ }^{\circ} \mathrm{C}$ to $27^{\circ} \mathrm{C}$ incrementally reduce photobody number by stimulating phyB-FP disassembly from selective thermo-unstable photobodies. The thermostability of photobodies relies on phyB's photosensory module. Surprisingly, elevated temperatures inflict opposite effects on phyB's functions in the hypocotyl and cotyledon despite inducing similar photobody dynamics, indicative of tissue/organ-specific temperature signaling circuitry either downstream of photobody dynamics or independent of phyB. Our results thus provide direct cell biology evidence supporting an early temperature signaling mechanism via dynamic assembly/disassembly of individual photobodies possessing distinct thermostabilities.

\footnotetext{
${ }^{1}$ Department of Botany and Plant Sciences, Institute for Integrative Genome Biology, University of California, Riverside, CA 92521, USA. ${ }^{2}$ These authors contribute equally: Joseph Hahm, Keunhwa Kim. ${ }_{\text {email: meng.chen@ucr.edu }}$
} 
T emperature is a critical environmental cue influencing all aspects of plant development and growth. Besides the stress-tolerant responses induced by extreme freezing or scorching conditions $\mathrm{s}^{1-3}$, changes in the range of ambient growth temperatures $\left(12-27^{\circ} \mathrm{C}\right.$ for Arabidopsis ${ }^{4}$ ) can also dramatically alter the rate of shoot and root growth and thereby plant architecture ${ }^{5,6}$, adjust cell differentiation patterns and developmental transitions such as reproductive organ formation ${ }^{7-9}$, and modulate plant immunity ${ }^{10-12}$. These phenomena are collectively called thermomorphogenesis ${ }^{13,14}$. Increases in global temperature have already had major impacts on plant phenology, distribution, diversity, and are expected to significantly decrease crop productivity ${ }^{15,16}$. Therefore, a molecular understanding of how plants sense and respond to temperature has become critical to the development of strategies for sustaining crop production in a changing climate ${ }^{17}$.

Plants monitor changes in ambient temperature by the red (R) and far-red (FR) photoreceptor, phytochrome $\mathrm{B}(\mathrm{phyB})^{18,19}$. PhyB is a bilin-containing pigment or a biliprotein that senses light using a covalently linked phytochromobilin as the chromophore ${ }^{20-22}$. Light induces the isomerization of the C15-C16 carbon-carbon double bond of phytochromobilin between a C15-Z (trans) and a C15-E (cis) configurations, and whereby, photoconverts phyB between two relatively stable conformations: an inactive, $\mathrm{R}$ light-absorbing $\operatorname{Pr}(\mathrm{C} 15-Z)$ and an active, FR light-absorbing Pfr $(C 15-E)^{20-22}$. The photoconversion of phyB, through changes in distinct ratios between active Pfr to inactive $\mathrm{Pr}$, allows plants to detect changes in light quality, quantity, and periodicity, thereby sensing the diurnal and seasonal time as well as the physical environment. For example, the existence of neighboring plants or vegetative shade enriches FR light and shifts the phyB equilibrium towards its inactive $\mathrm{Pr}$ form to induce the shade avoidance response ${ }^{23-25}$. The $\mathrm{Pfr} / \mathrm{Pr}$ equilibrium of phyB can also be influenced by temperature ${ }^{18}$. The active Pfr of phyB is thermally unstable and can spontaneously revert back to the inactive $\operatorname{Pr}$ in a light-independent process called dark or thermal reversion $20,26,27$. The thermal reversion of phyB is rapid and can be greatly enhanced by increases in temperature between 10 and $30^{\circ} \mathrm{C}^{18}$. This temperature-dependent, intrinsic property makes phyB a thermosensor of ambient temperature ${ }^{18,19}$. Therefore, temperature signaling by phyB is thought to be mediated by a similar mechanism as that of shade ${ }^{14,18,19}$.

Ambient temperature signaling is best studied in Arabidopsis (Arabidopsis thaliana) by examining how temperature modulates phyB-mediated light responses ${ }^{13,14}$. PhyB exerts prominent roles in photomorphogenesis during Arabidopsis seedling development, wherein photoactivation of phyB triggers differential organ growth of the embryonic stem (hypocotyl) and the embryonic leaf (cotyledon) ${ }^{28}$ - that is, while phyB promotes cotyledon expansion, it restricts hypocotyl elongation ${ }^{29,30}$. Hypocotyl elongation has been widely used as a readout for measuring phyB activity. Exposing Arabidopsis seedlings to warm temperatures (e.g., a transition from 21 to $27^{\circ} \mathrm{C}$ ) results in enhanced hypocotyl elongation akin to shade treatments ${ }^{13,14,31}$. In both scenarios, shifting phyB's equilibrium towards the inactive Pr releases its inhibitory effects on the stability and activity of PhytochromeInteracting Factors, the PIFs, which are a small group of nodal basic/helix-loop-helix transcriptional regulators promoting plant growth through the activation of genes involved in the biosynthesis and signaling of the plant growth hormone auxin and other hormones ${ }^{13,14,32}$. In contrast to hypocotyl elongation, cotyledon (and also leaf) expansion is promoted by phyB ${ }^{29}$. The current model of phyB's inhibitory role on the growth-promoting PIFs does not offer an intuitive explanation for phyB-mediated cotyledon expansion. Nonetheless, seedlings exposed to shade conditions display smaller cotyledons, which is consistent with the idea that shade inhibits phyB activity ${ }^{33}$. The temperature effect on phyB-mediated cotyledon expansion has not been explicitly described.

One of the earliest light responses at the cellular level-by either activation or inactivation of phyB-is the alteration of phyB's subcellular distribution ${ }^{34}$. Photoactivation of phyB promotes its assembly to subnuclear compartments named photobodies (PBs), while inactivation of phyB triggers its disassembly from $\mathrm{PBs}^{28,35-37}$. The light-dependent phyB assembly to $\mathrm{PBs}$ has been extensively studied using Arabidopsis transgenic lines expressing functional phyB tagged with a fluorescent protein (phyB-FP). PhyB-FPs are synthesized in the dark as the Pr form in the cytoplasm. During the dark-to-light transition, when seedlings emerge from the soil and encounter light, photoactivated phyB-FPs translocate from the cytoplasm to the nucleus and initially localize to many small foci, and several hours later, to a few large foci referred hereafter as $\mathrm{PBs}^{36-38}$. Conversely, during the light-to-dark transition, the inactivation of phyB-FP by dark/ thermal reversion stimulates its redistribution from PBs to small foci and the nucleoplasm ${ }^{39}$. The steady-state pattern of phyB-FP in continuous light also alters with changes in light quantity and quality (i.e., R/FR ratio) and correlates tightly with phyBmediated regulation of hypocotyl growth in Arabidopsis ${ }^{40}$. Previous studies of the steady-state $\mathrm{PB}$ dynamics by molecular genetics and computational modeling support the notion that $\mathrm{PB}$ localization requires the $\mathrm{Pfr}^{36,40-43}$. Strong light conditions, which stabilize the Pfr, promote the assembly of phyB-FP PBs of $0.7-2 \mu \mathrm{m}$ in diameter ${ }^{40,44}$. By contrast, shade conditions and dim light, which enhance the conversion to the $\mathrm{Pr}$, lead to the relocalization of phyB-FP from PBs to tens of small foci of 0.1-0.7 $\mu \mathrm{m}$ in diameter and the nucleoplasm ${ }^{40,45}$. Molecular genetic studies on mutants with altered $\mathrm{PB}$ patterns indicate that $\mathrm{PBs}$ are required for phyB signaling $44,46-53$.

Changes in ambient temperature can also alter the morphology of PBs. It has been shown that transitions from $20^{\circ} \mathrm{C}$ to different temperatures between 10 and $30^{\circ} \mathrm{C}$ altered the size of PBs quadratically-that is, $\mathrm{PBs}$ were the largest at $20^{\circ} \mathrm{C}$ and became smaller in either cooler or warmer temperatures ${ }^{18}$. However, the current data do not explain why PBs responded to temperature quadratically. It is also unclear, since warm temperatures act similarly as shade ${ }^{18,19}$, whether warm temperatures induce similar PB dynamics as shade. Moreover, because phyB triggers differential responses in hypocotyl and cotyledon growth, it remains unknown whether temperature induces different $\mathrm{PB}$ dynamics in early phyB signaling in the hypocotyl and cotyledon. To address these questions, we characterized the steady-state PB patterns of phyB-FP in epidermal cells from the hypocotyl and cotyledon of two Arabidopsis ecotype backgrounds, Col-0 and Ler, in four ambient growth temperatures, $12,16,21$ and $27^{\circ} \mathrm{C}$. We show that increases in temperature from 12 to $27^{\circ} \mathrm{C}$ progressively reduce the number of PBs in all cell types by stimulating phyB-FP disassembly from selective thermo-unstable $\mathrm{PBs}$, while thermostable $\mathrm{PBs}$ persist even at $27^{\circ} \mathrm{C}$. Although the PB formation is mediated by phyB's Cterminal module, the thermostability of PBs depends on phyB's $\mathrm{N}$-terminal photosensory module. Surprisingly, cotyledon and hypocotyl cells display similar PB dynamics despite opposing temperature effects in phyB signaling, indicating tissue/organspecific temperature signaling mechanisms downstream of $\mathrm{PB}$ dynamics. These results thus demonstrate that temperature induces largely different $\mathrm{PB}$ dynamics than those by shade in early phyB signaling, thereby providing direct cell biology evidence supporting a unique temperature signaling mechanism via dynamic assembly and disassembly of individual PBs with distinct thermostabilities. 


\section{Results}

Tissue/organ-specific temperature effects on phyB signaling. $\mathrm{PBs}$ have been characterized extensively using transgenic lines expressing phyB-FP36,39,40,44-46,54,55, and therefore to examine the dynamics of phyB-FP PBs induced by temperature changes, we went back to the $p h y B-F P$ lines, including $P B G$ ( $p h y B-G F P$ ), which expresses functional phyB-GFP to complement the null phyB-5 mutant in the Landsberg erecta (Ler) background ${ }^{36}$, and $P B C$ (phyB-CFP), which expresses phyB-CFP to complement the null phyB-9 mutant in Col- $0^{46,55}$. Because both $P B G$ and $P B C$ overexpress phyB-FP 36,55 , we first examined whether they retain the same temperature effects as their respective ecotype background. In response to temperature increases from $12{ }^{\circ} \mathrm{C}$ to $27^{\circ} \mathrm{C}$, Ler and Col- 0 seedlings grown in continuous $10 \mu \mathrm{mol} \mathrm{m}^{-2} \mathrm{~s}^{-1} \mathrm{R}$ light exhibited gradual increases in hypocotyl length in a 2.4- and 4.5 -fold dynamic range, respectively (Fig. 1a,b) ${ }^{56} . P B G$ and $P B C$ lines showed reduced hypocotyl responses with a 1.4-fold dynamic range for both lines. However, they retained similar temperature effects on phyB signaling as Ler and Col-0, respectively_that is, warmer temperatures attenuated the function of phyB or enhanced hypocotyl elongation in all four lines (Fig. 1a, b). The reduced temperature responses in hypocotyl elongation in $P B G$ and $P B C$ are likely due to overexpression of phyB-FPs. Increasing the flux of phyB signaling in Ler and Col-0 did not lead to a similar effect as overexpression of phyB-FPs. For example, Ler and Col-0 seedlings grown in a higher light intensity, $50 \mu \mathrm{mol} \mathrm{m}^{-2} \mathrm{~s}^{-1} \mathrm{R}$ light (R50), showed even greater dynamic ranges of the temperature response (Fig. 1a, b), suggesting that the dynamic range of the temperature response in hypocotyl elongation relies on the level of phyB. Together, these results indicate that $P B G$ and $P B C$ maintain a reduced but similar warm temperature-dependent antagonistic effect on phyBdependent inhibition of hypocotyl elongation as Ler and Col-0, respectively.

We next examined the effect of temperature on cotyledon expansion. Seedlings of a variety of Arabidopsis ecotypes, including Ler and Col-0, accelerate cotyledon expansion when moved from low intensity (i.e., below $10 \mu \mathrm{mol} \mathrm{m}{ }^{-2} \mathrm{~s}^{-1}$ ) to high intensity (i.e., above $50 \mu \mathrm{mol} \mathrm{m}^{-2} \mathrm{~s}^{-1}$ ) of $\mathrm{R}$ light, and this cotyledon response depends on phyB $^{29,57}$. Therefore, in contrast to restricting hypocotyl growth, phyB promotes light-dependent cotyledon expansion ${ }^{29}$. To our surprise, warmer temperatures further enhanced phyB-dependent cotyledon expansion in Ler and Col- 0 in permissive $50 \mu \mathrm{mol} \mathrm{m}{ }^{-2} \mathrm{~s}^{-1} \mathrm{R}$ light (Fig. 1c, d). We did not observe a linear trend for the temperature effects on cotyledon expansion in $10 \mu \mathrm{mol} \mathrm{m}^{-2} \mathrm{~s}^{-1} \mathrm{R}$ light, despite a slight reduction of cotyledon size from 16 to $27^{\circ} \mathrm{C}$ (Fig. 1c, d). Interestingly, $P B G$ and $P B C$ grown in $10 \mu \mathrm{mol} \mathrm{m}^{-2} \mathrm{~s}^{-1} \mathrm{R}$ light also showed increases in cotyledon area with temperature in a similar manner as their respective ecotype-background lines grown in $50 \mu \mathrm{mol} \mathrm{m}^{-2} \mathrm{~s}^{-1} \mathrm{R}$ light (Fig. 1c, d). These results suggest that the high levels of phyB-FP in $P B G$ and $P B C$ lines could compensate for the low-light conditions and enable the temperature-dependent cotyledon expansion response. Therefore, we conclude that $P B G$ and $P B C$ retain the same temperature effects in promoting phyB-dependent cotyledon expansion as Ler and Col-0, respectively.

Temperature-dependent PB dynamics in the hypocotyl. To investigate how ambient temperature influences $\mathrm{PB}$ morphology, we first characterized the steady-state patterns of phyB-GFP PBs in epidermal-cell nuclei of the top one-third of the hypocotyl in 4-day-old $P B G$ seedlings grown at $12,16,21$, and $27^{\circ} \mathrm{C}$. We chose to grow seedlings in $10 \mu \mathrm{mol} \mathrm{m} \mathrm{m}^{-2} \mathrm{~s}^{-1} \mathrm{R}$ light because it has been previously shown that in this light condition phyB-FP localizes only to PBs (as opposed to some small foci as well) ${ }^{40,55}$. We quantified the number and volume of $\mathrm{PBs}$ using threedimensional imaging analysis (see Methods). To our surprise, we did not observe a shade-like transition from $\mathrm{PB}$ to small foci with temperature increases. Instead, phyB-GFP localizes to PBs in all four temperatures (Fig. 2a, top panels). Intriguingly, the average number of PBs per nucleus decreased progressively with temperature increases from four-to-five at $12^{\circ} \mathrm{C}$ to two-to-three at $27^{\circ} \mathrm{C}$ (Fig. 2b). We found that PBs can be categorized into two types based on their relative positions to the nucleolus: one type at the nucleolar periphery, referred hereafter as nucleolarassociated $\mathrm{PB}$ (Nuo-PB), and the other type away from the nucleolus, referred hereafter as non-nucleolar-associated $\mathrm{PB}$ (nonNuo-PB). The numbers of both Nuo-PBs and nonNuo-PBs decreased with temperature increases (Fig. 2b). The average number of Nuo-PBs per nucleus dropped from two at $12{ }^{\circ} \mathrm{C}$ to one at $27^{\circ} \mathrm{C}$ (Fig. 2a, b). Similarly, the number of nonNuo-PBs declined from two-to-three per nucleus at $12{ }^{\circ} \mathrm{C}$ to one per nucleus at $27^{\circ} \mathrm{C}$ (Fig. 2a, b). Consistently, the percentage of nuclei containing two or more either Nuo-PBs or nonNuo-PBs reduced gradually with temperature increases (Fig. 2c, d). For instance, at $12{ }^{\circ} \mathrm{C}, 87 \pm 3 \%$ of the nuclei had two or more nonNuo-PBs, whereas at $27^{\circ} \mathrm{C}$, the majority of the nuclei $(67 \pm 5 \%)$ had either only one or zero nonNuo-PB (Fig. 2a, d). The volume of PB became larger with temperature increases (Fig. 2e). Interestingly, the amount of phyB-GFP in $P B G$ increased with temperature and was more than tripled at $27^{\circ} \mathrm{C}$ compared to that at $12^{\circ} \mathrm{C}$ (Fig. 2f). Therefore, the increases in $\mathrm{PB}$ size with temperature could be due to the redistribution of phyB-GFP to fewer PBs and/or the enhanced phyB accumulation. Because the expression of $p h y B$ GFP was under the control of the $35 \mathrm{~S}$ constitutive promoter ${ }^{36}$, the accumulation of phyB-GFP is likely due to a mechanism at the post-transcriptional level. Together, these results show that increases in temperature progressively reduce the number of PBs in the nuclei of hypocotyl epidermal cells. Not all PBs reacted to temperature equally, while some thermo-unstable PBs disappeared in warmer temperatures, certain $\mathrm{PBs}$ appear to be thermostable even at $27^{\circ} \mathrm{C}$.

To test whether the temperature-dependent $\mathrm{PB}$ dynamics vary in different Arabidopsis ecotypes, we characterized the PBs in the $P B C$ line. Similar to $P B G$, the numbers of total, Nuo, and nonNuo PBs in the nuclei of hypocotyl epidermal cells in $P B C$ decreased with temperature increases (Fig. 2a, b). The percentage of nuclei containing two or more either Nuo-PBs or nonNuo-PBs declined with temperature increases (Fig. 2a-d). The PBs also became larger with temperature increases in $P B C$, so as the level of phyBCFP (Fig. 2e, f). The main difference between $P B C$ and $P B G$ was that $P B C$ had significantly more PBs per nucleus than $P B G$. This difference was particularly obvious in lower temperatures. For example, at $12^{\circ} \mathrm{C}$, while $P B G$ contained four to five total $P B s$ per nucleus, $P B C$ had almost twice as many (Fig. 2a, b). Interestingly, while the numbers of Nuo-PBs of $P B G$ and $P B C$ differed only slightly, the number of nonNuo-PBs per nucleus in $P B C$ was more than doubled compared with that in $P B G$-that is, two to three nonNuo-PBs in $P B G$ vs. six to eight in $P B C$ (Fig. 2a, b). Therefore, the differences in $P B$ numbers between $P B C$ and $P B G$ were mainly contributed by the numbers of nonNuo-PBs. The differences in $P B$ number between $P B G$ and $P B C$ were unlikely caused by a difference in the amount of phyB-FP because the levels of phyB-GFP and phyB-CFP are comparable between these two lines across the temperature range examined (Fig. 2f). Intriguingly, the dynamic changes in $\mathrm{PB}$ number by temperature were also mainly contributed by the variations in nonNuo-PBs (Fig. 2b), suggesting that nonNuo-PBs are more dynamically regulated by both environmental and genetic factors. Together, these results show that temperature changes trigger similar PB 
a

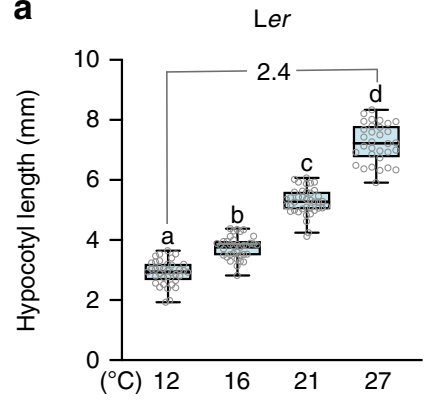

b

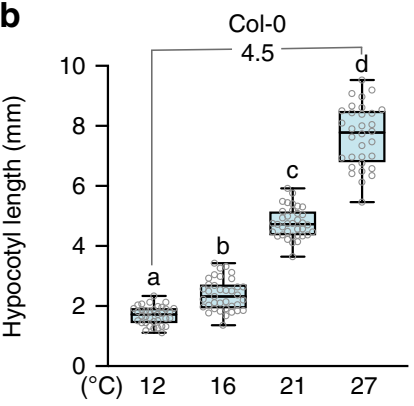

C

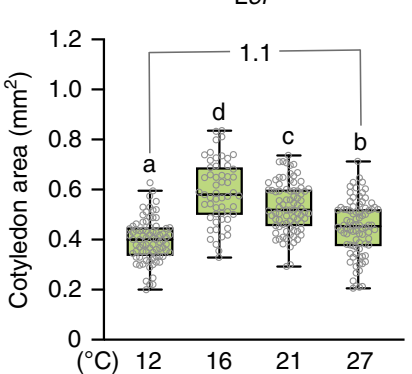

d

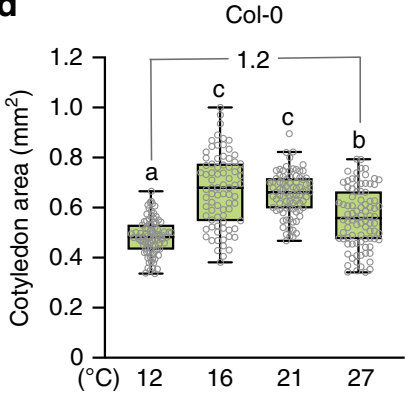

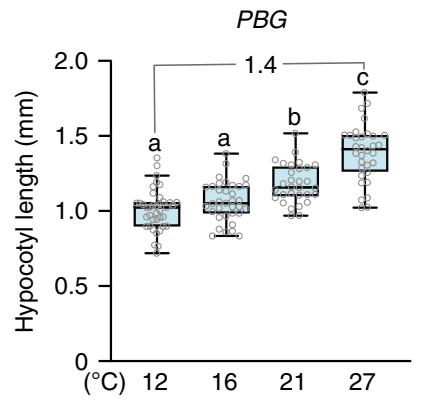
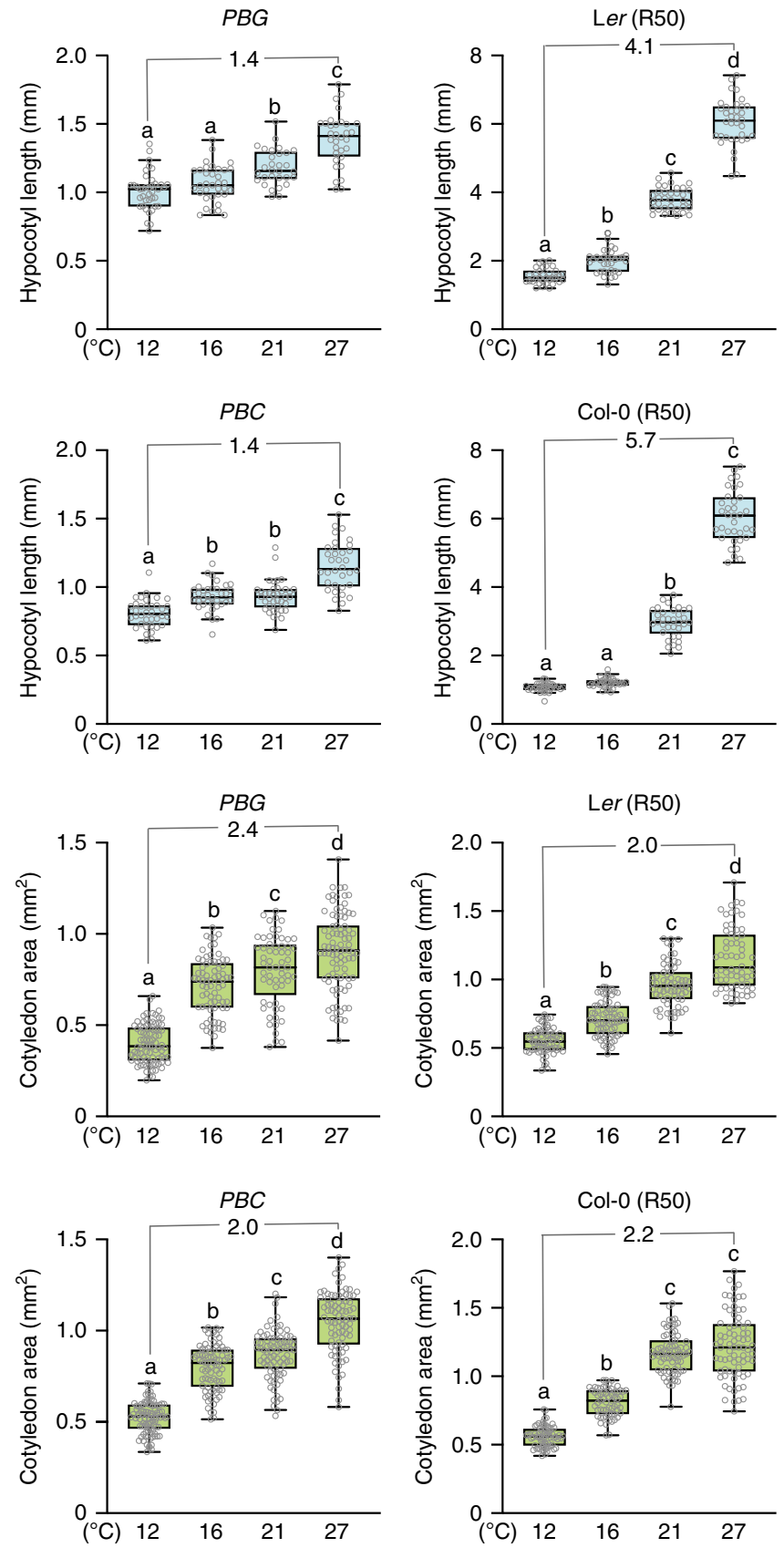

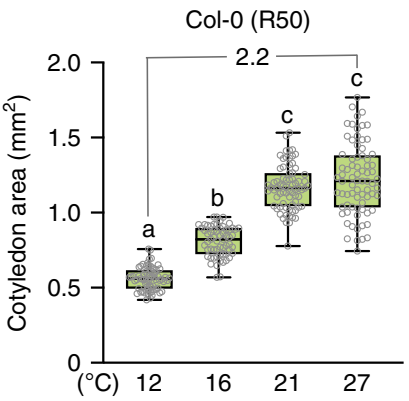

Fig. 1 Temperature imposes opposite effects on phyB functions in hypocotyl and cotyledon. $\mathbf{a}, \mathbf{b}$ Warmer temperatures attenuate the function of phy $B$ signaling in inhibiting hypocotyl growth. Hypocotyl length measurements of 4-day-old seedlings of Ler and PBG (a) and Col-0 and PBC (b) grown under 10 or 50 (R50) $\mu \mathrm{mol} \mathrm{m}{ }^{-2} \mathrm{~s}^{-1} \mathrm{R}$ light at $12,16,21$, and $27^{\circ} \mathrm{C}$. c, d Warmer temperatures enhance the function of phyB signaling in promoting cotyledon expansion. Cotyledon size measurements of 4-day-old seedlings of Ler and PBG (a) and Col-0 and PBC (b) grown under 10 or 50 (R50) $\mu$ mol $\mathrm{m}^{-2} \mathrm{~s}^{-1}$ $\mathrm{R}$ light at $12,16,21$, and $27^{\circ} \mathrm{C}$. For $\mathbf{a}-\mathbf{d}$, the fold changes of the median values of either hypocotyl length or cotyledon area between 27 and $12{ }^{\circ} \mathrm{C}$ are shown. For the box and whisker plots, the boxes represent from the 25th to the 75th percentile and the bars equal the median values. Samples with different letters exhibit statistically significant differences (ANOVA, Tukey's HSD, $P \leq 0.05, n \geq 33$ ). The source data underlying the hypocotyl (a, b) and cotyledon (c, d) measurements are provided in the Source Data file.

dynamics in the hypocotyl epidermal cells in Ler and Col, despite variations in the precise number of PBs per nucleus between the two ecotypes. These results suggest that individual PBs vary in thermostability, and temperature changes trigger dynamic assembly/disassembly of selective thermo-unstable PBs, while thermostable PBs can persist even in elevated temperatures.

Temperature-dependent PB dynamics in the cotyledon. $\mathrm{PB}$ dynamics have been characterized mainly in epidermal cells of the hypocotyl. It remains unclear whether PB dynamics are influenced by tissue/organ type. Given temperature elicits opposing effects on the function of phyB signaling in hypocotyl elongation and cotyledon expansion, we asked whether temperature induces similar or distinct PB dynamics in the cotyledon. To that end, we characterized the steady-state PB patterns under the same series of ambient temperatures in the epidermal cells on the adaxial side of the cotyledon. Interestingly, similar to hypocotyl epidermal cells, temperature increases from 12 to $27^{\circ} \mathrm{C}$ led 
a
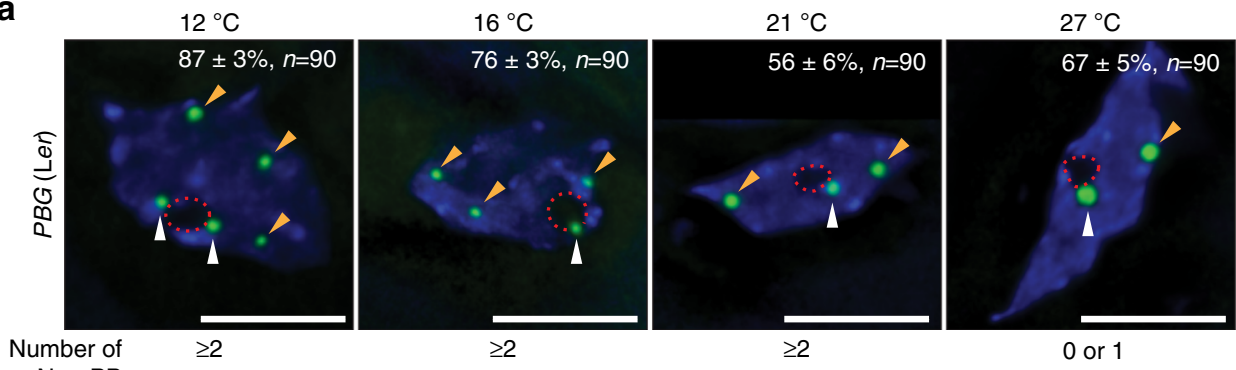

nonNuo-PBs
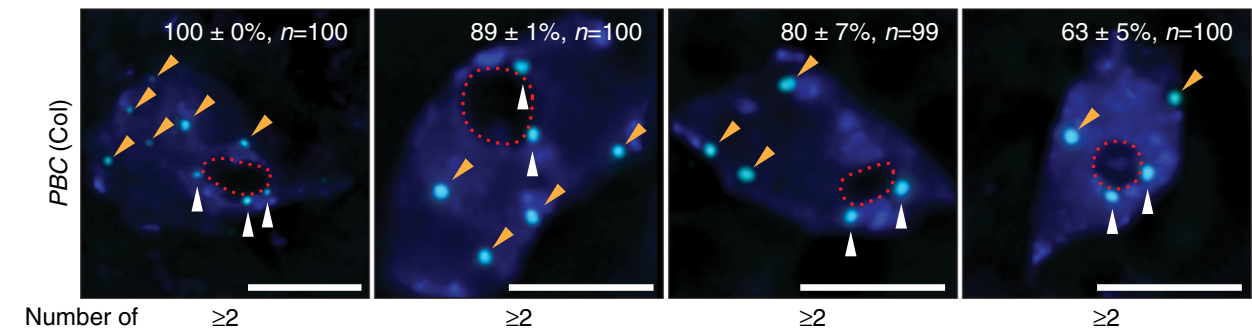

nonNuo-PBs

b
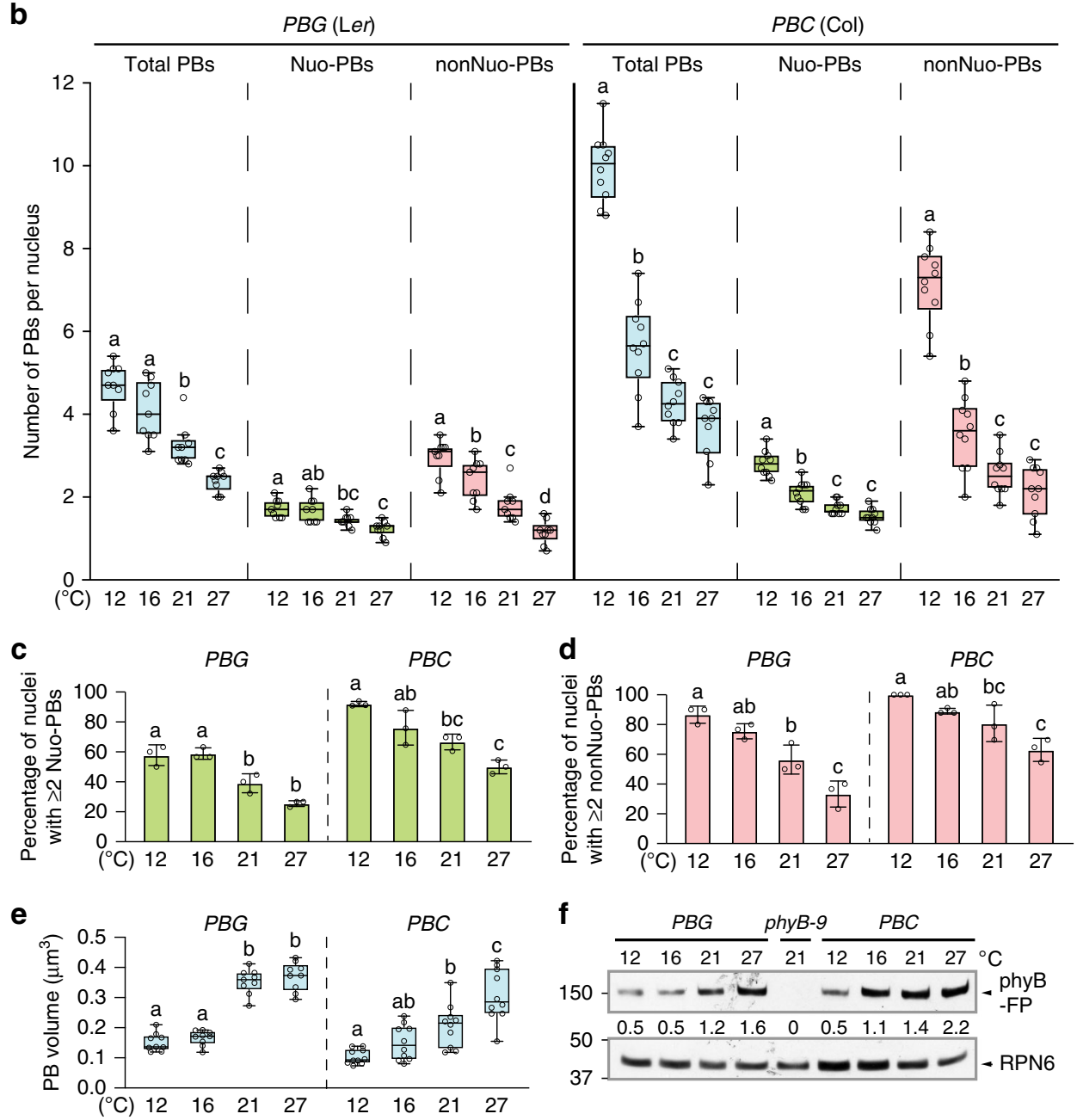

f

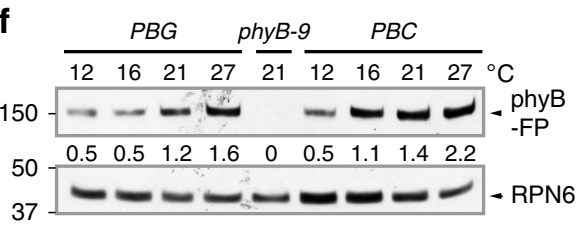

to progressive decreases in the total number of $\mathrm{PBs}$ in the cotyledon epidermal nuclei in both $P B G$ and $P B C$ (Fig. 3a-c). Notably, the cotyledon nuclei contained fewer PBs compared with hypocotyl nuclei. For example, the cotyledon nuclei in $P B G$ grown at $12{ }^{\circ} \mathrm{C}$ had mostly two-to-three PBs vs. four-to-five PBs in the hypocotyl nuclei (Figs. $2 \mathrm{~b}$ and $3 \mathrm{~b}$ ). Similarly, the cotyledon nuclei of $P B C$ grown at $12{ }^{\circ} \mathrm{C}$ contained five-to-six $\mathrm{PBs}$ compared

to nine-to-ten PBs in the hypocotyl nuclei (Figs. $2 \mathrm{~b}$ and $3 \mathrm{c}$ ). In $P B C$, the numbers of both Nuo-PBs and nonNuo-PBs in cotyledon cells also declined with temperature increases (Fig. 3c); the percentage of nuclei with two or more of either Nuo-PBs or nonNuo-PBs decreased dramatically from 12 to $27^{\circ} \mathrm{C}$ (Fig. 3a, d). Comparing the $\mathrm{PB}$ numbers between $P B G$ and $P B C$, we found that the cotyledon epidermal nuclei in $P B C$ had more $P B s$ than 
Fig. 2 Temperature-dependent photobody dynamics in hypocotyl epidermal cells. a Maximum-projection, deconvolved fluorescence microscopy images showing representative steady-state patterns of phyB-GFP (green) or phyB-CFP (cyan) PBs in hypocotyl epidermal-cell nuclei of 4-day-old PBG or PBC seedlings, respectively, grown under $10 \mu \mathrm{mol} \mathrm{m} \mathrm{m}^{-2} \mathrm{~s}^{-1} \mathrm{R}$ light at $12,16,21^{\circ} \mathrm{C}$, and $27^{\circ} \mathrm{C}$. Nuclei were labeled by DAPI (blue), and the boundaries of the nucleoli are traced by red dashed lines. Nuo-PBs and nonNuo-PBs are indicated by white and orange arrowheads, respectively. The percentage of nuclei including s.e. with the indicated PB pattern is shown in each image; $n$ indicates the total number of nuclei analyzed. Scale bars represent $5 \mu$ m.

b Quantification of the numbers of total, Nuo, and nonNuo PBs per nucleus in hypocotyl epidermal-cell nuclei of the PBG and PBC seedlings described in a. c Quantification of the percentage of nuclei in hypocotyl epidermal-cell nuclei of the $P B G$ and $P B C$ seedlings described in a with two or more Nuo-PBs. d Quantification of the percentage of nuclei in hypocotyl epidermal-cell nuclei of the PBG and PBC seedlings described in a with two or more nonNuo-PBs. For $\mathbf{c}$, d, error bars represent s.e. calculated from groups of seedlings. Different letters denote statistically significant differences (ANOVA, Tukey's HSD, $P \leq 0.05, n=3$ ). e Quantification of the volume of PBs in hypocotyl epidermal-cell nuclei of the PBG and PBC seedlings described in $\mathbf{a}$. For $\mathbf{b}, \mathbf{e}$, the boxes represent from the 25 th to the 75 th percentile and the bars equal the median values. Samples with different letters exhibit statistically significant differences (ANOVA, Tukey's HSD, $P \leq 0.05, n \geq 9$ ). $\mathbf{f}$ Immunoblot results showing the levels of phyB-GFP or phyB-CFP in 4-day-old PBG or PBC seedlings grown under $10 \mu \mathrm{mol} \mathrm{m} \mathrm{m}^{-2} \mathrm{~s}^{-1} \mathrm{R}$ light in the indicated temperatures. phyB-FPs were detected by anti-phyB antibodies. RPN6 was used as a loading control, and phyB-9 was used as a negative control. The relative levels of phyB-FPs, normalized against the corresponding levels of RPN6, are shown below the phyB-FP immunoblot. The source data underlying the PB measurements in $\mathbf{b}-\mathbf{e}$, and the immunoblots in $\mathbf{f}$ are provided in the Source Data file.

those in $P B G$ (Fig. 3a-c), this trend is the same as the hypocotyl (Fig. 2a, b). Interestingly, we found that the majority of the nuclei in $P B G$ cotyledon epidermal nuclei contained only two $\mathrm{PBs}$-one Nuo-PB and one nonNuo-PB-at $12{ }^{\circ} \mathrm{C}(68 \pm 3 \%), 16^{\circ} \mathrm{C}(64 \pm$ $3 \%)$, and $21^{\circ} \mathrm{C}(63 \pm 3 \%)$ (Fig. 3a, e). In striking contrast, at $27^{\circ} \mathrm{C}$, the nonNuo-PB disappeared in $67 \pm 3 \%$ of the cotyledon epidermal nuclei in $P B G$, leaving only one Nuo-PB (Fig. 3a, b, e). More than $85 \%$ of cotyledon epidermal nuclei in $P B G$ maintained one Nuo-PB across the temperature range between 12 and $27^{\circ} \mathrm{C}$ (Fig. 3e), indicating distinct thermostabilities between the thermosensitive nonNuo-PB and the thermo-insensitive Nuo-PB. The reduction of $\mathrm{PB}$ number with temperature increases suggests that the thermostability of PBs is determined by the stability of the Pfr form of phyB, which is attenuated by warmer temperatures ${ }^{18}$. Consistent with this hypothesis, increasing light intensity from 10 to $50 \mu \mathrm{mol} \mathrm{m}{ }^{-2} \mathrm{~s}^{-1} \mathrm{R}$ light, which stabilizes the Pfr, increased the numbers of $\mathrm{PBs}$ in the cotyledon cells of both $P B G$ and $P B C$ lines at $27^{\circ} \mathrm{C}$ (Fig. 3a-d). Similar to hypocotyl PBs, the size of the cotyledon PBs also increased with temperature increases (Fig. 3f). Together, these results further support the notion that individual PBs exhibit distinct thermostabilities. The number of PBs can be influenced by tissue/organ-specific factors. The fact that cotyledon and hypocotyl epidermal cells display similar PB dynamics suggests that the opposing effects of temperature on phyB signaling might be caused by tissue/organ-specific signaling circuitry downstream of PB dynamics or independent of phyB.

Temperature-induced rapid disassembly of thermosensitive PBs. The simplicity of the PB pattern in the $P B G$ cotyledon epidermal nuclei allows us to distinguish the two PBs based on their positions to the nucleolus, and therefore provides an opportunity to determine the kinetics of the disappearance of the thermosensitive nonNuo-PB during the 21 to $27^{\circ} \mathrm{C}$ transition. A major challenge in determining $\mathrm{PB}$ dynamics is that the localization pattern of phyB-FPs can be rapidly and dramatically altered by the excitation light during live-cell fluorescence imaging $^{58}$. To circumvent this problem, we fixed the seedling samples and characterized the steady-state phyB-GFP $\mathrm{PB}$ patterns at selected time points within $12 \mathrm{~h}$ after the 21 to $27^{\circ} \mathrm{C}$ transition (Fig. 4a). The results show that the percentage of nuclei containing one nonNuo-PB dropped from about $60 \%$ at $21^{\circ} \mathrm{C}$ at time 0 to $36 \%$ after $6 \mathrm{~h}$ at $27^{\circ} \mathrm{C}$ and maintained at this percentage for the rest of the time course (Fig. $4 \mathrm{a}, \mathrm{b}$ ). In contrast, the percentage of nuclei containing one Nuo-PB did not change (Fig. 4c). Neither the level of phyB-GFP nor the size of PBs varied more than 2 -fold (Fig. 4d, e). Together, these results demonstrate that individual $\mathrm{PBs}$ could respond to a specific temperature range and temperature increases induce rapid disassembly of phyB-GFP from distinct thermosensitive PBs.

phyB's C-terminal module localizes to thermo-insensitive PBs. The structural basis of the light-dependent $\mathrm{PB}$ localization of phyB has been extensively investigated. PhyB is a homodimer; $\mathrm{PB}$ localization requires the dimeric Pfr form of phyB $40,41,43,46,59$. Each phyB monomer contains an N-terminal photosensory module and a C-terminal output module ${ }^{20,21}$. PB localization of phyB is mediated by the dimeric form of phyB's C-terminal output module, which presumably contains localization signals or sequences for targeting phyB to the nucleus and $\mathrm{PBs}^{46,54,55}$. PhyB's C-terminal module alone localizes constitutively to $\mathrm{PBs}^{54,55}$. The current model posits that the subcellular targeting activities of the C-terminal output module is masked in the $\mathrm{Pr}$ form by the N-terminal module, at least in part, through lightdependent interactions between the two modules, the interaction between $\mathrm{N}$ - and C-terminal modules weakens in the Pfr and thereby the $\mathrm{PB}$ localization activity of the $\mathrm{C}$-terminal module is unmasked or exposed ${ }^{55}$. If this model is correct, the C-terminal module alone would be expected to localize to PBs that are temperature insensitive and should show a similar pattern to that of active phyB. To test this hypothesis, we used the BCY line, which expresses the phyB C-terminal module fused with YFP (BCY) in the phyB-9 background ${ }^{55}$, and tested whether the localization of BCY to PBs in cotyledon-cell nuclei in the dark can be altered by temperature changes between 12 and $27^{\circ} \mathrm{C}$. Supporting our hypothesis, the BCY-containing $\mathrm{PBs}$ did not respond to temperature changes. In all four temperatures tested from 12 to $27^{\circ} \mathrm{C}$, most of the nuclei contained more than eight PBs, including at least two Nuo-PBs and six nonNuo-PBs (Fig. 5a, b). The percentage of nuclei with such a PB pattern stayed the same across the temperature range (Fig. 5d). Interestingly, comparing the $\mathrm{PB}$ pattern of $B C Y$ with that of $P B C$ (both in the phyB-9 background), the pattern of BCY PBs resembles that of PBC PBs at $12^{\circ} \mathrm{C}$, where the Pfr is more stabilized (Fig. $3 \mathrm{a}, \mathrm{c}$ ). The number of BCY PBs, including Nuo-PBs and nonNuo-PBs, also stayed the same between dark- and light-grown $B C Y$ lines (Fig. 5a, b, d). These results indicate that the localization of BCY to PBs is not responsive to light and temperature, and also is not influenced by possible heterodimerization with other phys in the light ${ }^{60,61}$. Consistent with the thermo-insensitivity of BCY PBs, the cotyledon size of $B C Y$ did not respond to temperature changes in the dark (Fig. 5e). The sizes of dark-grown $B C Y$ cotyledons were at least 5 -fold smaller than those of $P B C$ grown in the light, suggesting that phyB's C-terminal module alone is not sufficient in promoting cotyledon expansion (Figs. $1 \mathrm{~d}$ and $5 \mathrm{e}$ ). $B C Y$ seedlings grown in the light did show significantly larger cotyledons 
a
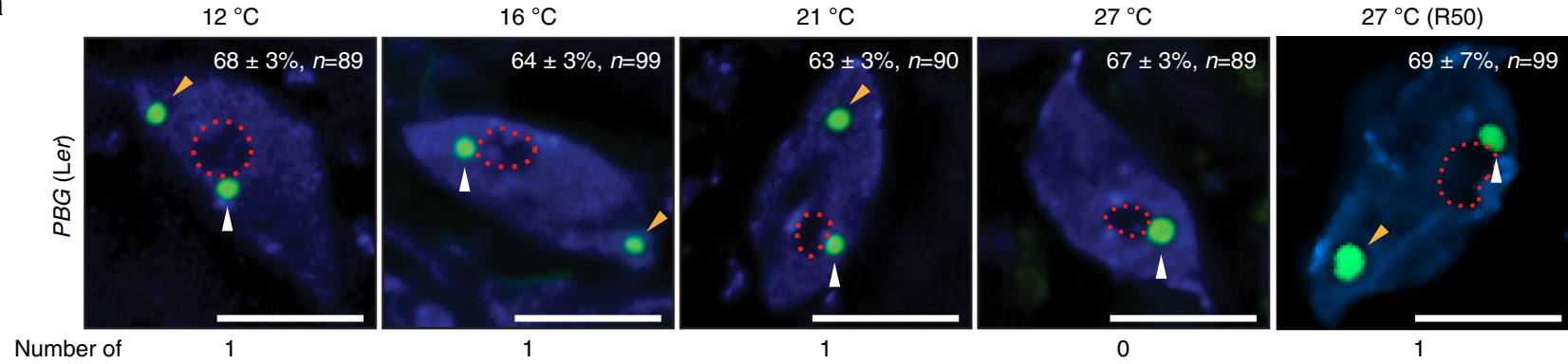

nonNuo-PBs
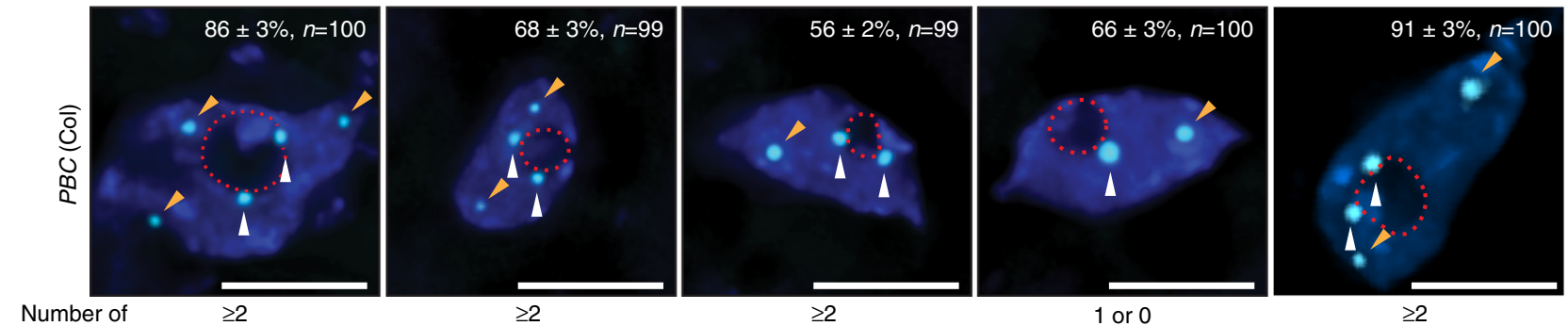

nonNuo-PBs

b

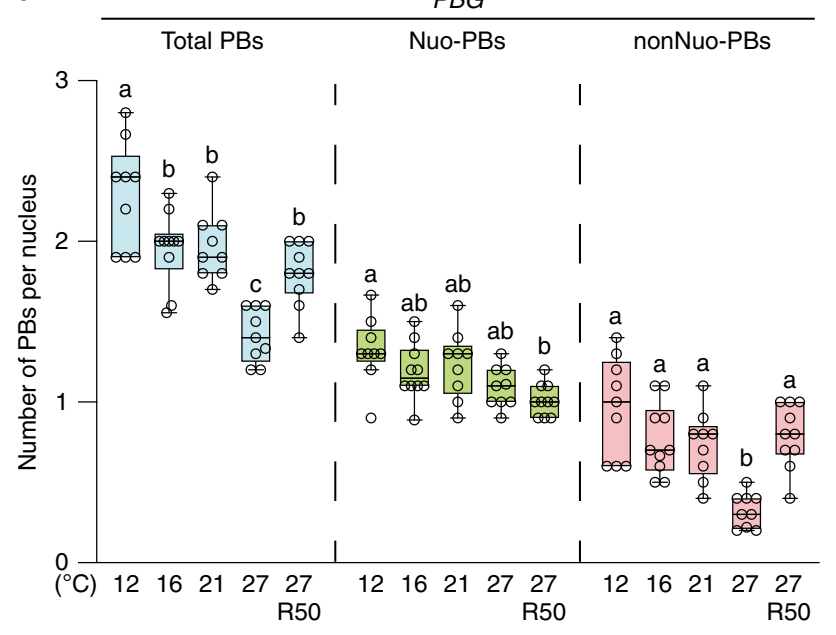

C

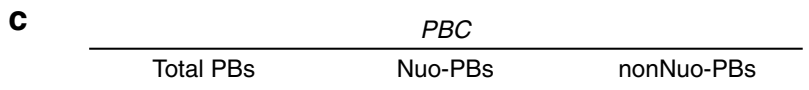

d

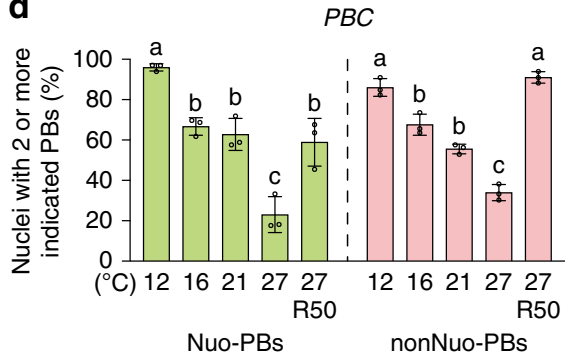

e

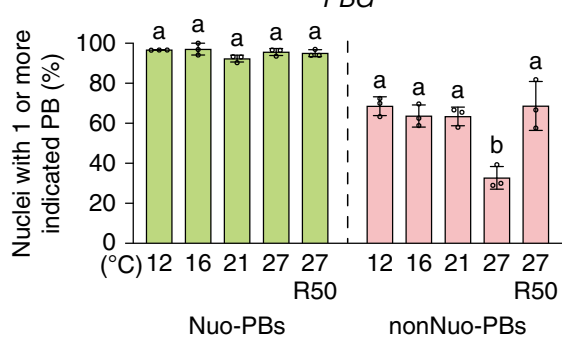

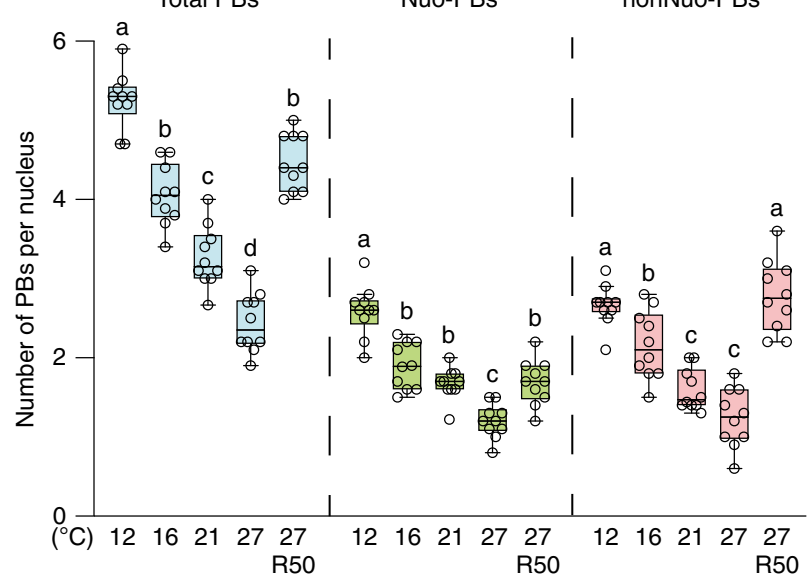

f

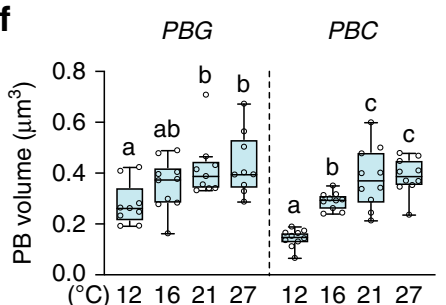

Fig. 3 Temperature-dependent photobody dynamics in cotyledon epidermal cells. a Maximum-projection, deconvolved fluorescence microscopy images showing representative steady-state patterns of phyB-GFP (green) or phyB-CFP (cyan) PBs in cotyledon epidermal-cell nuclei of 4 -day-old PBG or PBC

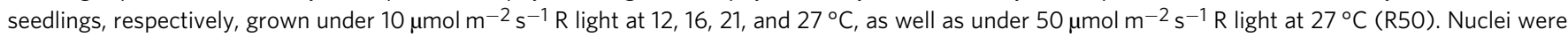
labeled by DAPI (blue), and the boundaries of nucleoli are traced by red dashed lines. Nuo-PBs and nonNuo-PBs are indicated by white and orange arrowheads, respectively. The percentage (with s.e.) of nuclei with the indicated PB pattern is shown in each image; $n$ indicates the total number of nuclei analyzed. Scale bars represent $5 \mu \mathrm{m}$. b, c Quantification of the numbers of total, Nuo, and nonNuo PBs per nucleus in cotyledon epidermal-cell nuclei of the $P B G$ (b) and PBC (c) seedlings described in a. d Quantification of the percentages of nuclei in cotyledon epidermal-cell nuclei with two or more either Nuoor nonNuo-PBs in the PBC seedlings described in a. e Quantification of the percentage of nuclei in cotyledon epidermal-cell nuclei containing one or more either Nuo- or nonNuo-PB in the PBG seedlings described in a. For d, e, error bars represent s.e. calculated from groups of seedlings. Different letters denote statistically significant differences (ANOVA, Tukey's HSD, $P \leq 0.05, n=3$ ). $\mathbf{f}$ Quantification of the volume of PBs in cotyledon epidermal-cell nuclei of the PBG and PBC seedlings described in $\mathbf{a}$. For $\mathbf{b}, \mathbf{c}, \mathbf{f}$, the boxes represent from the 25 th to the 75 th percentile, and the bars equal the median values. Samples with different letters exhibit statistically significant differences in PB number (ANOVA, Tukey's HSD, $P \leq 0.05, n \geq 9$ ). The source data underlying the PB measurements in $\mathbf{b}-\mathbf{f}$ are provided in the Source Data file. 
a

$27^{\circ} \mathrm{C}$

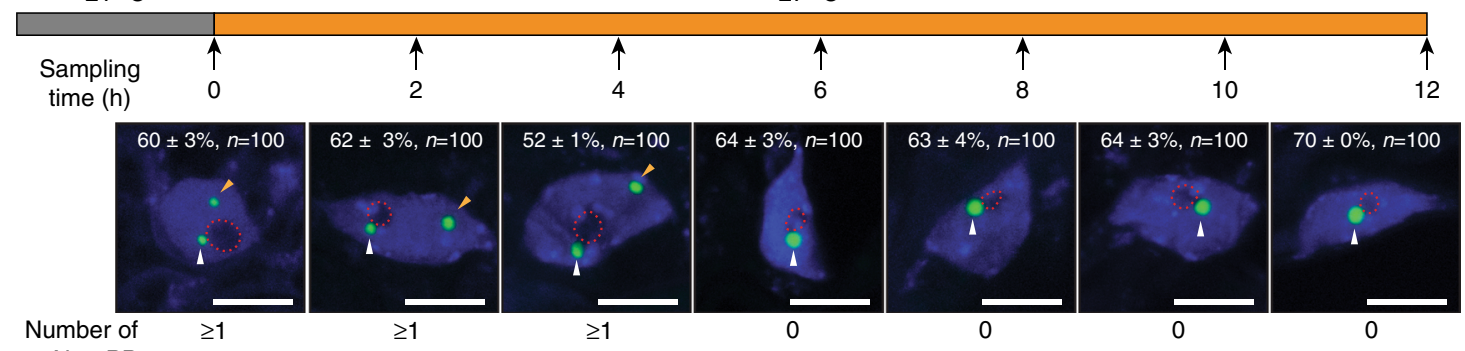

nonNuo-PBs

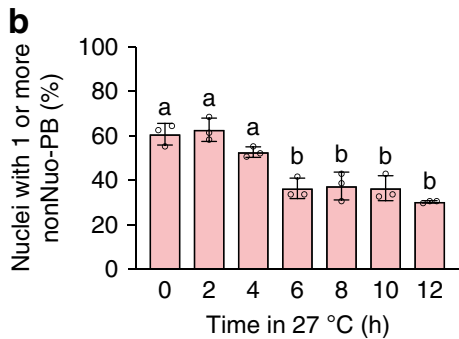

d

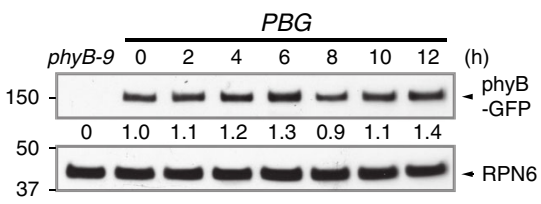

C
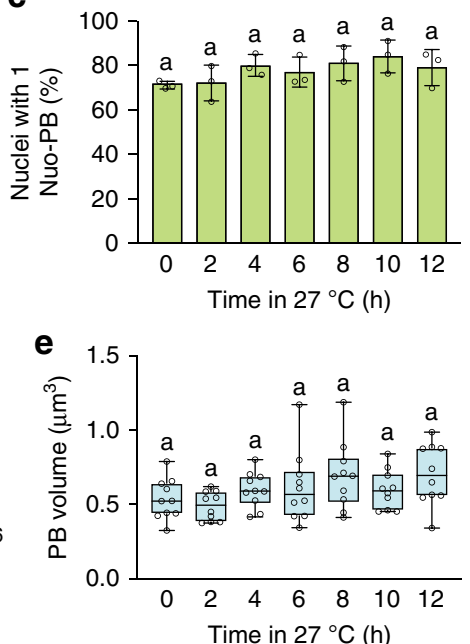

Fig. 4 Kinetics of warm temperature-induced disappearance of the nonNuo-PB in PBG. a Schematic illustration of the 21 to $27^{\circ} \mathrm{C}$ transition experiment with maximum-projection, fluorescence microscopy images showing representative steady-state patterns of phyB-GFP PBs in cotyledon epidermal-cell nuclei of 4-day-old PBG seedlings at the indicated time points. Nuclei were labeled by DAPI (blue), and the boundaries of the nucleoli are traced by red dashed lines. Nuo-PBs and nonNuo-PBs are indicated by white and orange arrowheads, respectively. The percentage of nuclei with s.e. showing the indicated PB pattern is included in each image; $n$ indicates the total number of nuclei analyzed. Scale bars represent $5 \mu \mathrm{m}$. $\mathbf{b}$ Quantification of the percentage of cotyledon epidermal-cell nuclei with one or more nonNuo-PB in the PBG seedlings described in a. c Quantification of the percentage cotyledon epidermal-cell nuclei with one Nuo-PB in the $P B G$ seedlings described in $\mathbf{a}$. For $\mathbf{b}$, c, error bars represent s.e. calculated from groups of seedlings. Different letters denote statistically significant differences (ANOVA, Tukey's HSD, $P \leq 0.05, n=3$ ). $\mathbf{d}$ Immunoblot results showing the levels of phyB-GFP in 4-day-old PBG seedlings grown under $10 \mu \mathrm{mol} \mathrm{m} \mathrm{m}^{-2} \mathrm{~s}^{-1} \mathrm{R}$ light in the indicated time points during the 21 to $27^{\circ} \mathrm{C}$ transition. phyB-GFP was detected by anti-phyB antibodies, and RPN6 was used as a loading control. phyB-9 was used as a negative control. The relative levels of phyB-GFP normalized against the corresponding levels of RPN6 are shown below the phyB-GFP immunoblot. e Quantification of the volume of PBs in cotyledon epidermal-cell nuclei of the $P B G$ seedlings during the 21 to $27^{\circ} \mathrm{C}$ transition described in $\mathbf{a}$. The boxes represent from the 25th to the 75th percentile, and the bars equal the median values. Samples with the same letter exhibit no statistically significant difference in PB volume (ANOVA, Tukey's HSD, $P \leq 0.05, n=10$ ). The source data underlying the PB measurements in $\mathbf{b}, \mathbf{c}, \mathbf{e}$, and the immunoblots in $\mathbf{d}$ are provided in the Source Data file.

compared with dark-grown $B C Y$ seedlings (Fig. 5e), which is likely due to the actions of other phys. The level of BCY also increased with temperature increases (Fig. 5f). However, in this case, the volume of individual BCY PBs did not change significantly (Fig. $5 \mathrm{~g}$ ), possibly due to the distribution of the additional BCY to the large numbers of PBs. Together, these results indicate that temperature does not affect the $\mathrm{PB}$ localization activity of the C-terminal module of phyB per se, and therefore the thermostability of PBs likely depends on phyB's N-terminal photosensory module ${ }^{18}$.

YHB preferentially localizes to one thermo-insensitive $\mathrm{PB}$. To further examine the structural basis of PB's thermostability, we turned to the constitutively active phyB mutant YHB, which carries a $\mathrm{Y} 276 \mathrm{H}$ mutation in phyB's chromophore attachment domain and locks phyB in an active form ${ }^{53}$. It is important to note that although YHB is biologically active, it represents a unique active conformation because YHB is poorly photoactive and stuck mainly in an R light-absorbing conformation-that is, a biologically active $\mathrm{Pr}$ form ${ }^{53,62-64}$. Although YHB has been shown to localize constitutively to PBs even in the $\operatorname{dark}^{50,53,65}$, the pattern of YHB PBs has not been characterized in comparison with the wild-type phyB PBs. If the thermostability of $\mathrm{PBs}$ is determined by the $\mathrm{N}$-terminal module, a constitutively active phyB is expected to show a similar $\mathrm{PB}$ pattern as BCY or phyB-FP in low temperatures. To test this hypothesis, we used a YHB line, which expresses YHB-YFP in the phyB-9 background $^{66}$. The pattern of YHB-YFP PBs in cotyledon epidermal nuclei in the dark stayed the same between 12 and $27^{\circ} \mathrm{C}$ (Fig. 5a, c). However, this pattern was strikingly different from that of BCY. The majority of the nuclei contained only a YHBYFP Nuo-PB without any nonNuo-PB, and the rest nuclei contained one Nuo-PB and one nonNuo-PB (Fig. 5a, c). The pattern of YHB-YFP PBs is thus similar to that of phyB-CFP at $27^{\circ} \mathrm{C}$ with only thermostable PBs (Fig. 3a, c). The pattern of YHB-YFP PBs stayed the same between light- and dark-grown YHB seedlings (Fig. 5a, c-e). The level of YHB-YFP increased 


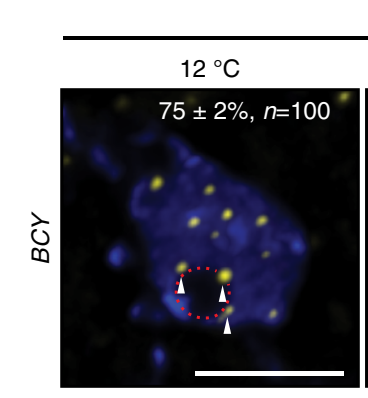

Dark

Red

\section{a}
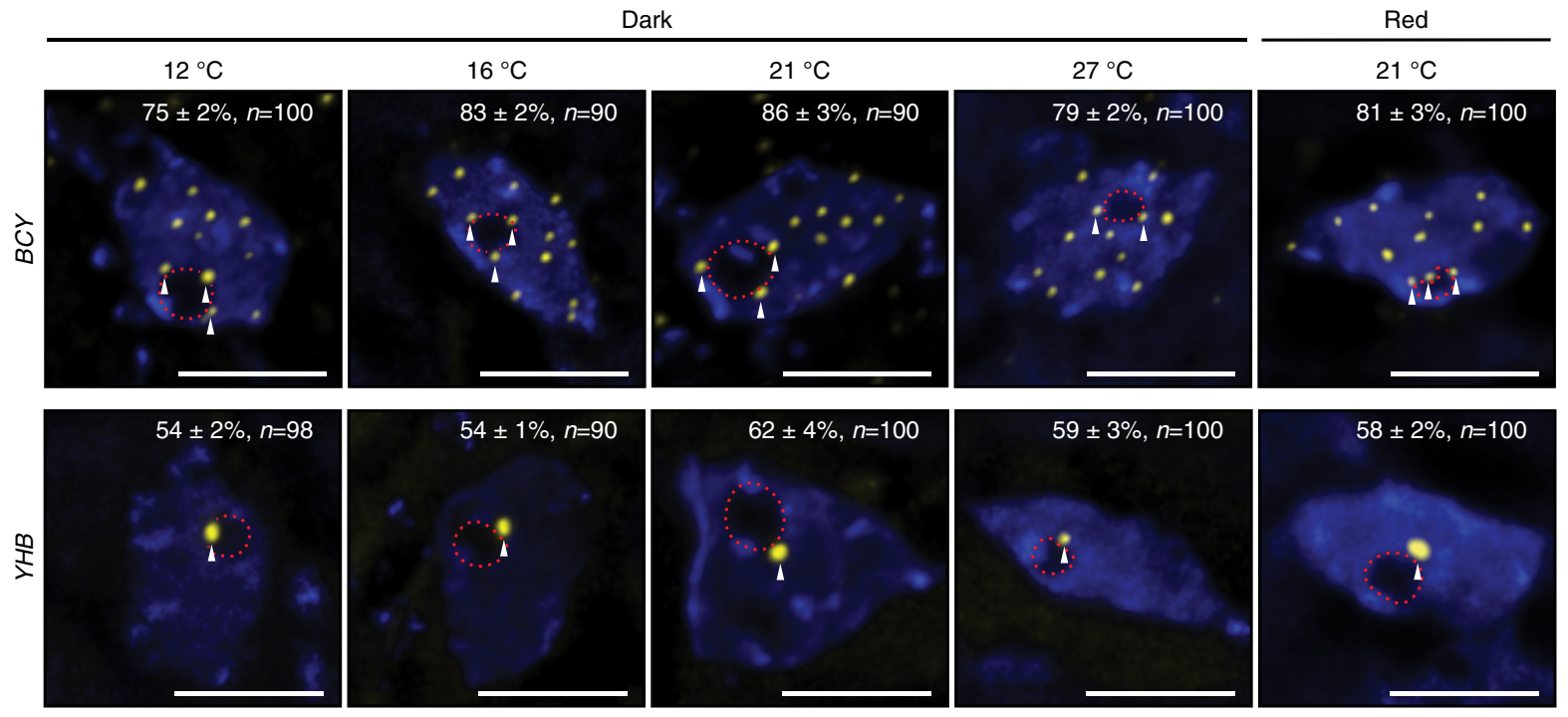

b

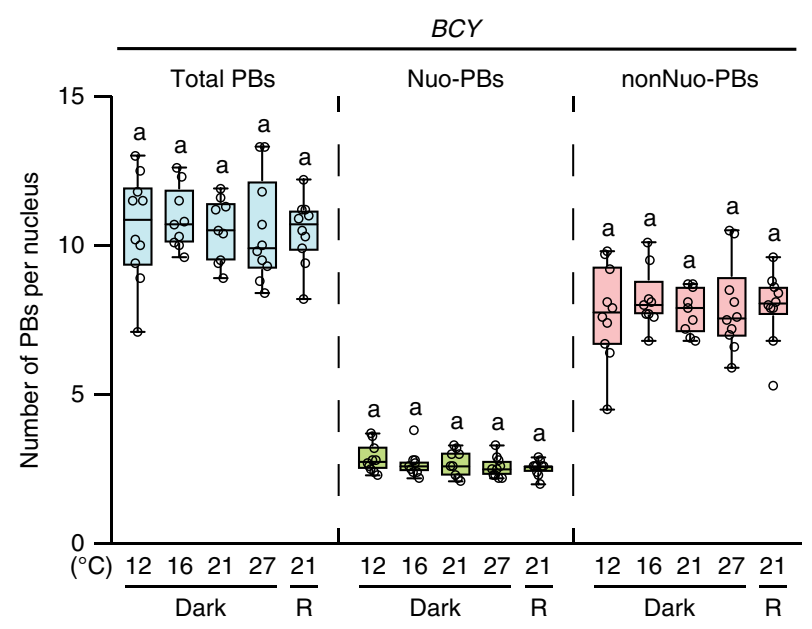

d

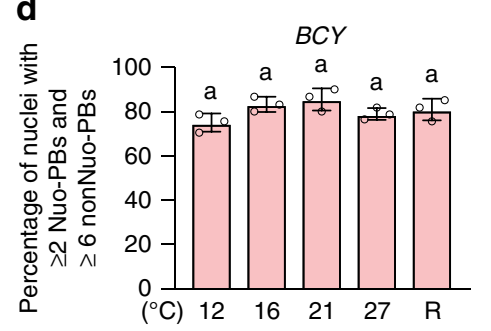

f

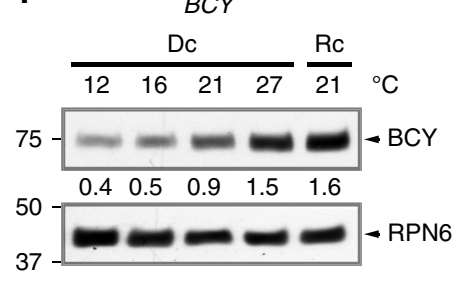

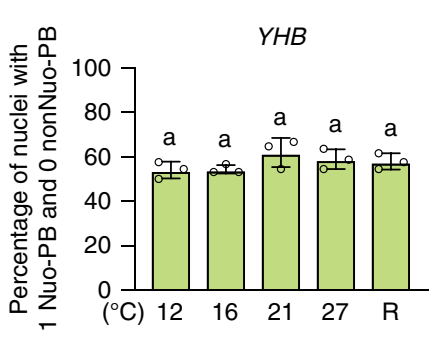

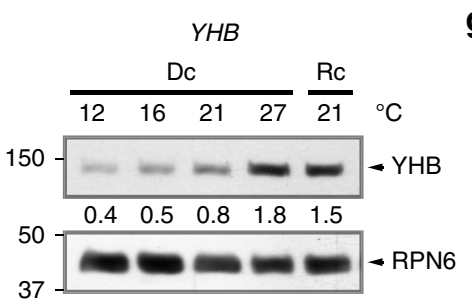

C

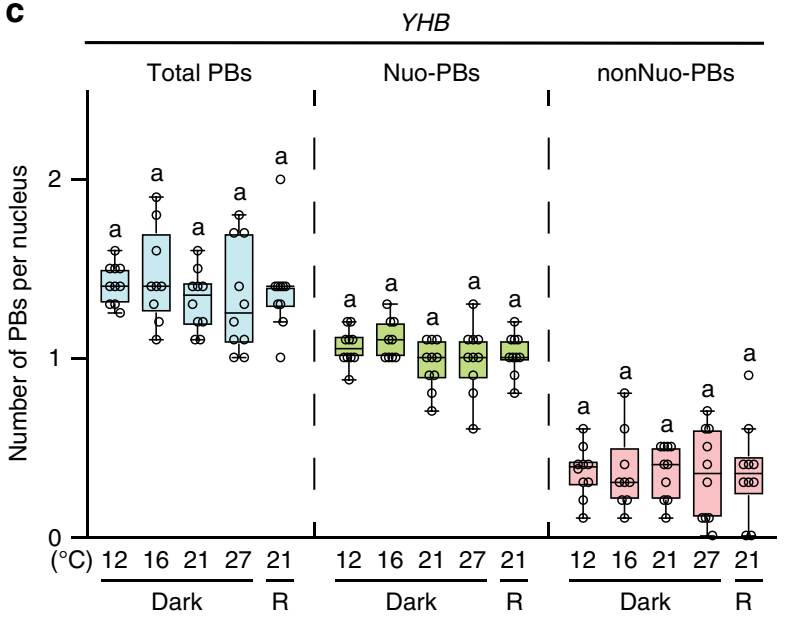

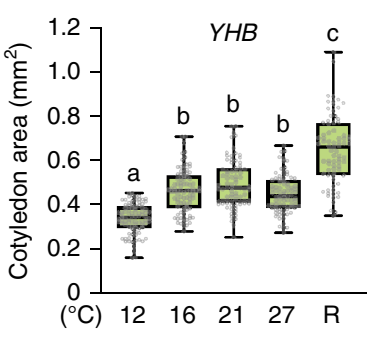

g

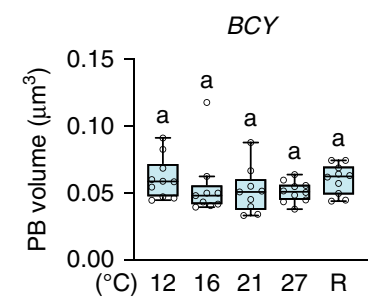

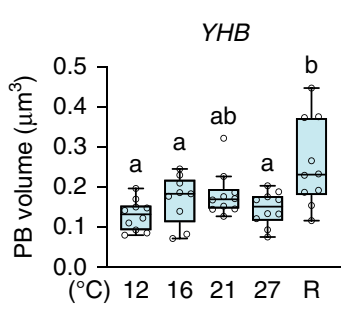

with temperature; in this case, we also observed an increase in the size of YHB-YFP PBs at $27^{\circ} \mathrm{C}$ (Fig. $5 \mathrm{f}, \mathrm{g}$ ). Together, these results show that disruption of the ability of conformational changes within the $\mathrm{N}$-terminal module of phyB abrogates the temperature responsiveness of $\mathrm{PBs}$, supporting the notion that the thermosensitivity of PBs relies on the N-terminal module.
The fact that YHB localizes preferentially to only a couple of thermostable PBs as opposed to multiple PBs like phyB-CFP suggests that either the assembly of thermosensitive PBs might require the photo or thermal reversion of phyB or the specific conformation of YHB only permits its assembly to selective thermostable PBs. 
Fig. 5 BCY and YHB-YFP display distinct temperature-insensitive PB patterns. a Maximum-projection, deconvolved images showing representative steady-state patterns of phyB C-terminal module fused with YFP (BCY) or YHB-YFP PBs in cotyledon epidermal-cell nuclei of 4-day-old BCY and YHB seedlings, respectively, grown at $12,16,21$, and $27^{\circ} \mathrm{C}$ in the dark or in $10 \mu \mathrm{mol} \mathrm{m} \mathrm{m}^{-2} \mathrm{~s}^{-1}$ in $\mathrm{R}$ light. Nuclei were labeled by DAPI (blue), white arrowheads indicate Nuo-PB, and the boundaries of the nucleoli are traced by red dashed lines. The percentage of nuclei with s.e. showing the representative PB pattern is shown in each image; $n$ indicates the total number of nuclei analyzed. Scale bars represent $5 \mu \mathrm{m}$. $\mathbf{b}$, c Quantification of the numbers of total, Nuo, and nonNuo PBs per nucleus in cotyledon epidermal-cell nuclei of the $B C Y(\mathbf{b})$ and $Y H B$ (c) seedlings described in a. $\mathbf{d}$ Quantification of the percentage of nuclei with the indicated number of PBs in cotyledon epidermal-cell nuclei of the $B C Y$ and $Y H B$ seedlings described in a. Error bars represent s.e. calculated from groups of seedlings. Different letters denote statistically significant differences (ANOVA, Tukey's HSD, $P \leq 0.05, n=3$ ). e Cotyledon size measurements of the $B C Y$ or $Y H B$ seedlings described in a. $\mathbf{f}$ Immunoblot results showing the levels of BCY or YHB-YFP in 4-day-old $B C Y$ or $Y H B$ seedlings grown in the dark or $10 \mu \mathrm{mol} \mathrm{m} \mathrm{m}^{-2} \mathrm{~s}^{-1} \mathrm{R}$ light in the indicated temperatures. BCY and YHB-YFP were detected by anti-phyB antibodies. RPN6 was used as a loading control. The relative levels of BCY and YHB-YFP normalized against the corresponding levels of RPN6 are shown. $\mathbf{g}$ Quantification of the volume of BCY and YHBYFP PBs in cotyledon epidermal-cell nuclei of the $B C Y$ and $Y H B$ seedlings described in $\mathbf{a}$. For $\mathbf{b}, \mathbf{c}, \mathbf{e}, \mathbf{g}$, the boxes represent from the 25th to the 75th percentile and the bars equal the median values. Samples with different letters exhibit statistically significant differences (ANOVA, Tukey's HSD, $P \leq 0.05$, for $\mathbf{b}, \mathbf{c}$, and $\mathbf{g}, n \geq 9$, for $\mathbf{e}, n \geq 78$ ). The source data underlying the PB measurements in $\mathbf{b}-\mathbf{d}, \mathbf{g}$, cotyledon measurements in $\mathbf{e}$, and immunoblots in $\mathbf{f}$ are provided in the Source Data file.

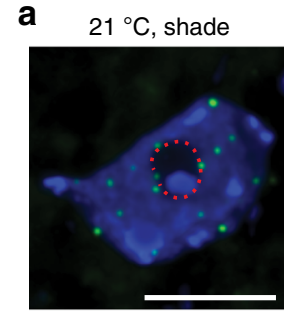

C 60
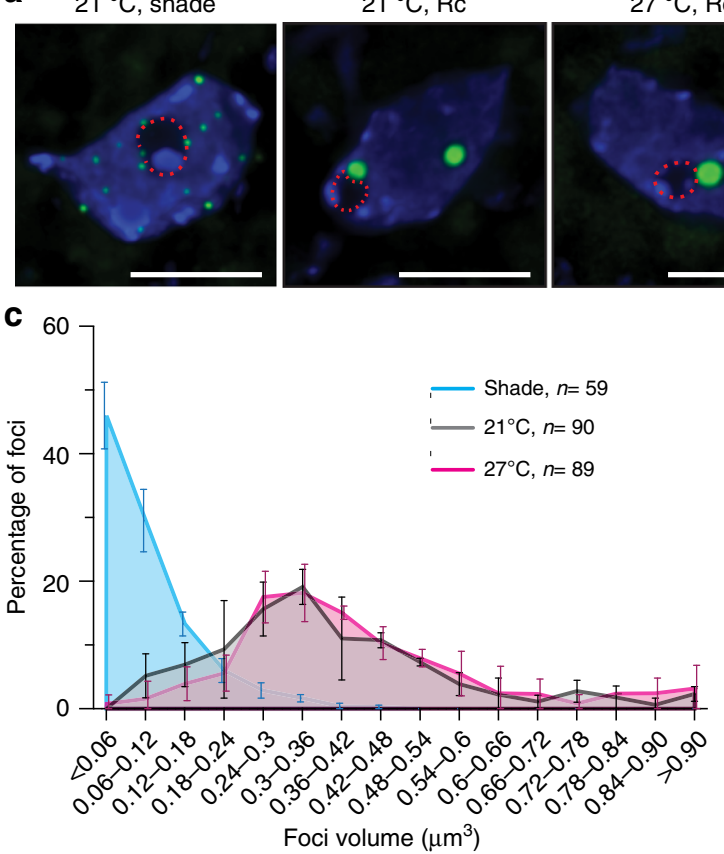

b
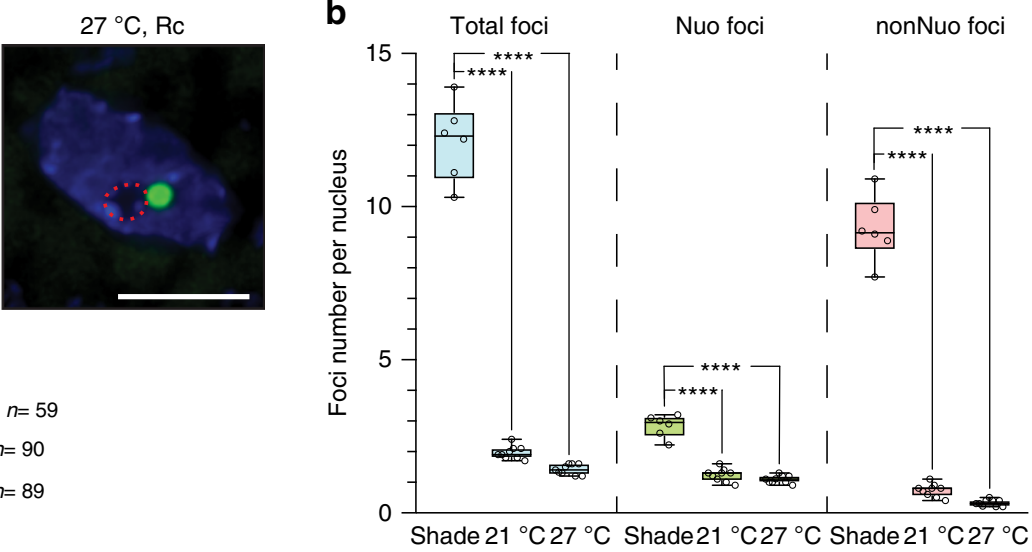

d

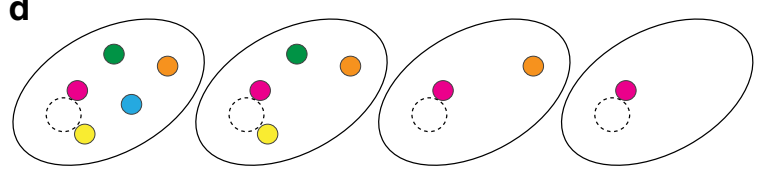

$12{ }^{\circ} \mathrm{C}$

$21^{\circ} \mathrm{C}$

$27^{\circ} \mathrm{C}$

Thermostability

Fig. 6 Warm temperature and shade elicit distinct photobody dynamics. a Maximum-projection, deconvolved fluorescence microscopy images showing representative steady-state patterns of phyB-GFP in cotyledon epidermal-cell nuclei of 4-day-old $P B G$ seedlings grown at $21^{\circ} \mathrm{C}$ in $10 \mu \mathrm{mol} \mathrm{m}^{-2} \mathrm{~s}^{-1}$ continuous $\mathrm{R}$ light $\left(21^{\circ} \mathrm{C}, \mathrm{Rc}\right)$, at $21^{\circ} \mathrm{C}$ in $10 \mu \mathrm{mol} \mathrm{m}-2 \mathrm{~s}^{-1} \mathrm{R}$ light supplemented with $10 \mu \mathrm{mol} \mathrm{m}-2 \mathrm{~s}^{-1} \mathrm{FR}$ light $\left(21^{\circ} \mathrm{C}\right.$, shade), and at $27^{\circ} \mathrm{C}$ in $10 \mu \mathrm{mol} \mathrm{m}^{-2} \mathrm{~s}^{-1}$ $\mathrm{R}$ light. $\mathbf{b}$ Quantification of the numbers of total, Nuo, and nonNuo PBs or foci per nucleus in cotyledon epidermal-cell nuclei of the PBG seedlings described in $\mathbf{a}$. The boxes represent from the 25th to the 75th percentile and the bars equal the median values. Asterisks represent statistically significant differences in PB number (unequal variances $t$ test, $P \leq 0.0001, n \geq 6$ ). c Distribution of the volume of PBs or foci in cotyledon epidermal-cell nuclei of the PBG seedlings grown in the indicated shade, $21^{\circ} \mathrm{C}$, and $27^{\circ} \mathrm{C}$ conditions described in a. Error bars represent s.d. $\mathbf{d}$ Schematic illustration of the model of temperature-dependent PB dynamics. The circles filled with different colors represent PBs with a gradient of distinct thermostabilities. The circle with a dotted line represents the nucleolus. The most thermostable PB persists in all temperatures, whereas the less thermostable ones form only in lower temperatures. As a result, temperature increases progressively trigger the disassembly of individual PBs. The source data underlying the PB measurements in $\mathbf{b}, \mathbf{c}$ are provided in the Source Data file.

Warm temperature and shade induce distinct PB dynamics. The current model suggests that warm temperature attenuates phyB functions by enhancing the thermal reversion of phyB to its inactive $\operatorname{Pr}$ form. Therefore, warm temperature signaling is thought to work in a similar manner as shade, which promotes the photoconversion of phyB to the Pr. Previous studies have shown that an increase in FR light under continuous $\mathrm{R}$ light or an end-of-day FR treatment leads to the relocalization of phyB-FP to many small foci and the nucleoplasm ${ }^{40}$. However, the temperature-dependent $\mathrm{PB}$ dynamics we observed are quite different from the published $\mathrm{PB}$ response by $\mathrm{FR}$ or shade. To confirm this discrepancy between warm temperature and shade in the same cell type, we characterized the localization pattern of phyB-GFP in cotyledon epidermal-cell nuclei of $P B G$ grown at 21 ${ }^{\circ} \mathrm{C}$ in a simulated shade condition with $10 \mu \mathrm{mol} \mathrm{m}^{-2} \mathrm{~s}^{-1} \mathrm{R}$ light supplemented by $10 \mu \mathrm{mol} \mathrm{m}{ }^{-2} \mathrm{~s}^{-1} \mathrm{FR}$ light. We found that all phyB-GFP-containing PBs disappeared under the shade condition, phyB-GFP localized to many smaller subnuclear foci (Fig. 6a-c). The numbers of both Nuo and nonNuo foci increased in the shade condition compared with the PBs in 10 $\mu \mathrm{mol} \mathrm{m} \mathrm{s}^{-2} \mathrm{R}$ light at 21 and $27^{\circ} \mathrm{C}$ (Fig. 6b). Because it is rather arbitrary to define large PBs and small foci, we looked at 
the distribution of the sizes of the PBs or foci in the three datasets (Fig. 6c). The results clearly show that the transition to small foci appeared unique to the shade response, as the size distribution of $\mathrm{PBs}$ remained the same between 21 and $27^{\circ} \mathrm{C}$ (Fig. 6c). These results, therefore, demonstrate that warm temperature and shade induce distinct PB dynamics.

\section{Discussion}

The assembly of phyB to PBs is one of the earliest cellular events in phyB signaling ${ }^{36,38}$, but how $\mathrm{PB}$ dynamics are involved in temperature sensing is less understood. Here we systematically examined the PB patterns in two Arabidopsis ecotypes, Col-0 and Ler, and in two tissue/organ types, hypocotyl and cotyledon, across the ambient temperature range from 12 to $27^{\circ} \mathrm{C}$. Our results demonstrate that increasing temperature triggers a linear response in $\mathrm{PB}$ number in all cells examined. Surprisingly, increases in temperature from 12 to $27^{\circ} \mathrm{C}$ elicit progressive disassembly of only selective temperature-sensitive or thermounstable PBs, while thermostable PBs persist even at warm temperatures, suggesting that individual PBs possess distinct thermostabilities and could serve as temperature sensors (Fig. 6d). We also defined two types of PBs, Nuo-PBs and nonNuo-PBs, and found that the dynamic changes in $\mathrm{PB}$ number by temperature, ecotype, and tissue/organ type are mainly contributed by changes in nonNuo-PBs, and that one of the Nuo-PBs is particularly thermostable. The thermostability of $\mathrm{PBs}$ relies on phyB's N-terminal photosensory module. The temperaturedependent $\mathrm{PB}$ dynamics reflect the enhanced thermal reversion of active phyB by warmer temperatures and correlate with the responsiveness of temperature-induced morphological changes, but unexpectedly, do not always agree with the effects of temperature on phyB signaling. For example, hypocotyl and cotyledon epidermal cells display similar temperature-dependent $\mathrm{PB}$ dynamics despite the opposing temperature effects on the function of phyB signaling in regulating hypocotyl and cotyledon growth, suggesting that the morphological effects of temperature could be determined by tissue/organ-specific signaling circuitry either downstream of $\mathrm{PB}$ dynamics or independent of phyB ${ }^{18}$. Together, our results unveil a unique early temperature signaling mechanism via the assembly/disassembly of individual thermosensitive PBs (Fig. 6d), which is largely different from the disappearance of all PBs induced by shade.

Our results revealed unexpectedly that individual PBs exhibit distinct thermostabilities, thereby sensitive to a specific temperature range (Fig. 6d). This conclusion is supported by the incremental decreases in $\mathrm{PB}$ number with temperature increases in the cotyledon and hypocotyl epidermal cells of both $P B G$ and $P B C$ seedlings (Figs. 2 and 3). Only a subset of $P B s$ disappeared with temperature increases, there was at least one Nuo-PB that could persist in warm temperatures in all the cells examined (Figs. 2-4). Even thermosensitive PBs did not disappear at once (Figs. 2 and 3). PB localization of phyB has been suggested to stabilize the Pfr form or attenuate thermal reversion ${ }^{39}$; our results therefore suggest that individual PBs might stabilize Pfr to different extents and thereby causing the distinct temperature requirements for phyB disassembly among $\mathrm{PBs}$ - that is, individual PBs carry distinct thermosensitivities (Fig. 6d). This phenomenon was best exemplified in the cotyledon epidermal nuclei of $P B G$, which contained mostly two PBs-a Nuo-PB and a nonNuo-PB-allowing us to distinguish and follow the responses of particular PBs (Fig. 3a, b). Strikingly, while the Nuo-PB was insensitive to the temperature range between 12 and $27^{\circ} \mathrm{C}$, the nonNuo-PB was sensitive to and became unstable specifically at $27^{\circ} \mathrm{C}$, and disappeared rapidly during the 21 to $27^{\circ} \mathrm{C}$ transition (Figs. 3a, b and 4). These results further support the conclusion that individual $\mathrm{PBs}$ carry distinct thermostabilities and can respond to a specific range of temperatures, thereby implying that individual PBs could act as temperature sensors (Fig. 6d). This model does not exclude the scenario in which the assembly of phyB into $\mathrm{PBs}$ requires a seeding or initiating mechanism that is temperature dependent.

We also observed that the volume of $\mathrm{PBs}$ increased with temperature increases in both hypocotyl and cotyledon cells in $P B C$ and $P B G$ (Figs. 2 and 3). This is likely due to a combination of reduction of $\mathrm{PB}$ number and increase in phyB levels (Figs. 2 and 3). It is important to note that at any given temperature, the sizes of $\mathrm{PBs}$ always fall into a relatively large range with significant variations (Figs. 2e and 3f), and therefore might not be sensitive enough to report a statistically significant change in $\mathrm{PB}$ size. For instance, the data did not show a significant change in $\mathrm{PB}$ volume during the 21 to $27^{\circ} \mathrm{C}$ transition in $P B G$ cotyledon cells (Fig. 4e), which could be due to the lack of resolution to recognize any small differences. We have consistently observed temperature-dependent increases in the level of phyB-FP in $P B C$, $P B G, Y H B$, and $B C Y$ (Figs. $2 \mathrm{f}$ and $5 \mathrm{f}$ ). Because these are transgenic lines overexpressing phyB-FPs, these results might suggest that the stability of phyB is enhanced by warmer temperatures and this property relies on phyB's C-terminal module. This is consistent with the idea that warmer temperatures enhance the thermal reversion of phyB from Pfr to Pr form, which is more stable ${ }^{67}$. However, further investigations are required to confirm this hypothesis.

Our results show that the number of $\mathrm{PBs}$ varies in different tissue/organ types and Arabidopsis ecotypes (Figs. 2b and 3b, c). Interestingly, the variations in $\mathrm{PB}$ number by tissue/organ type, ecotype, and temperature are mainly contributed by nonNuo-PBs (Figs. $2 \mathrm{~b}$ and $3 \mathrm{~b}, \mathrm{c}$ ). Therefore, it is tempting to speculate that the presence or absence of a $\mathrm{PB}$, particularly those nonNuo-PBs, could account for tissue/organ- and ecotype-specific responsiveness to temperature. This point needs to be validated by future investigations on the role of individual $\mathrm{PBs}$ in tissue/organ-type and ecotype-specific light and temperature responses at single-cell level. It is unclear what determines the number of PBs. One hypothesis is that PB number is controlled by the stability of PBs, alternatively, it could also be determined by a seeding mechanism for initiating $\mathrm{PB}$ assembly and this seedling mechanism can be influenced by ecotype-background and tissue/organ type. Our results reveal that warm temperatures impose opposing effects on the function of phyB signaling in the hypocotyl and cotyledon (Fig. 1). While it is well known that warm temperature attenuates the activity of phyB in inhibiting hypocotyl elongation, we found that it actually enhances the function of phyB signaling in promoting cotyledon expansion (Fig. 1). However, despite the opposite temperature effects, both hypocotyl and cotyledon cells showed similar PB dynamics, suggesting that the discrepancy in the temperature-dependent hypocotyl and cotyledon responses might be due to tissue/organ-specific signaling circuitry either downstream of PB dynamics or independent of phyB ${ }^{18}$. Consistent with this hypothesis, it has been shown that a group of auxin-responsive SAUR (Small Auxin Up RNA) genes respond differently to light signals between the hypocotyl and cotyledon ${ }^{68}$. It has also been shown that phyB promotes hypocotyl elongation at warm temperatures; however, our experiments were not particularly designed to test a correlation between PB dynamics and this particular response ${ }^{69}$. Notably, the temperature-induced PB dynamics observed in our study are different from the report by Legris et al. ${ }^{18}$ showing that the size of PBs peaks at $20^{\circ} \mathrm{C}$; instead, we observed continuous increases in PB volume from 12 to $27^{\circ} \mathrm{C}$. These discrepancies are likely attributable to the different experimental conditions and cell types used between the two studies. While we focused on the steady-state PB patterns in 
particular temperatures, in epidermal cells of the cotyledon and hypocotyl, and in seedlings grown in continuous monochromatic $\mathrm{R}$ light, the experiments by Legris et al. ${ }^{18}$ were carried out during temperature transitions, in epidermal and sub-epidermal cells of the hypocotyl, and in seedlings grown in diurnal white light conditions. As our results indicated that the PB number can be influenced largely by genetic and environmental variations, it is expected that different experimental settings could result in different PB patterns. Another possible reason for different observed $\mathrm{PB}$ patterns by temperature may be contributed by the different sample preparation techniques used in the two studies ${ }^{18}$. With that said, our main conclusion that temperature increases reduce the number of PBs is consistent with the decline in the Pfr level with temperature increases shown by the previous studies ${ }^{18,19}$.

What determines the thermostability of a PB and why individual PBs show different thermostabilities? To understand these questions, we need to examine the proposed kinetics of the photoconversion and thermal reversions between the phyB dimers of Pfr:Pfr and $\operatorname{Pr}: \operatorname{Pr}^{18,27,43}$. Activation or inactivation of the phyB dimer occurs in two steps: the conversion between $\operatorname{Pr}: \operatorname{Pr}$ homodimer and Pfr:Pr heterodimer and the conversion between Pfr:Pr heterodimer and Pfr:Pfr homodimer ${ }^{18,43}$. Both photoconversion and thermal reversions are expected to alter the $\mathrm{PB}$ localization of phyB. The thermal reversion from Pfr:Pfr to Pr:Pr involves two kinetic parameters: a slow Pfr:Pfr to Pfr:Pr reversion and a 100-fold faster Pfr:Pr to Pr:Pr reversion ${ }^{19,43}$. The current model posits that temperature influences phyB activity mainly through the conversion from Pfr:Pfr to Pfr:Pr in the dark or during nighttime, but through the conversion from Pfr:Pr to Pr: $\mathrm{Pr}$ in the light or during the daytime, because under illumination the Pfr:Pfr to Pfr:Pr conversion is significantly slower than the photoconversion rate from Pfr:Pr to Pfr:Pfr, and therefore can be neglected ${ }^{18,19}$ - that is, temperature indirectly affects Pfr:Pfr stability in the light ${ }^{18}$. Because PB localization requires the dimeric Pfr:Pfr form of $\mathrm{phyB}^{43,46}$, the thermostability of a $\mathrm{PB}$ is likely determined by the stability of the Pfr:Pfr dimers in the PB. Consistent with this notion, our results showed that the thermosensitivity of PBs is determined by the N-terminal photosensory module of phyB. Deleting (i.e., BCY) or mutating (i.e., $\mathrm{YHB})$ the photosensory module resulted in temperatureinsensitive PBs (Fig. 5). Moreover, increasing the intensity of light from 10 to $50 \mu \mathrm{mol} \mathrm{m}{ }^{-2} \mathrm{~s}^{-1} \mathrm{R}$ light, which stabilizes the Pfr: Pfr, promoted the assembly of more PBs at $27^{\circ} \mathrm{C}$ (Fig. 3a-e). The fact that $\mathrm{PBs}$ exhibit a range of thermostabilities in ambient temperature implies that the stability of the Pfr:Pfr of phyB is different among PBs. This could be due to different phyB binding partners or slightly different active phyB forms among individual PBs. This hypothesis is supported by the PB pattern of YHB. Despite being recognized as biologically active, YHB represents a unique phyB-active form that absorbs $\mathrm{R}$ light or still shares some characteristics of the Pr form ${ }^{53,62-64}$. Interestingly, YHB-YFP did not localize to as many $\mathrm{PBs}$ as phyB-FP or the C-terminal module of phyB (BCY); instead, it localized to only one or two PBs that resembles the thermostable $\mathrm{PBs}$ of phyB-CFP at $27^{\circ} \mathrm{C}$ (Fig. 5). These results support the idea that each $\mathrm{PB}$ might represent and could only accommodate a specific active-phyB state. The variation in thermostability or the Pfr:Pfr conformation among PBs could be due to differences in modifications of phyB itself and/or interactions with other $\mathrm{PB}$ constituents. For example, phosphorylation of phyB's N-terminal module can largely influence the stability and activity of the $\mathrm{Pfr}^{70-72}$. In particular, phosphorylation of Ser86 destabilizes the Pfr:Pfr form and attenuates PB localization ${ }^{70,72}$. Three phyB-interacting proteins have been reported to stabilize the Pfr form by suppressing its thermal reversion, they are ARABIDOPSIS RESPONSE REGULATOR 4 $(\text { ARR } 4)^{73}$, PHOTOPERIODIC CONTROL OF HYPOCOTYL 1
$(\mathrm{PCH} 1)^{50-52}$, and PCH1-LIKE (PCHL) ${ }^{52}$. In particular, PCH1 and PCHL facilitate the PB localization of phyB $^{50-52}$. Molecular genetic studies showed that $\mathrm{PB}$ localization of phyB requires a number of signaling components, including HMR (HEMERA) ${ }^{44,65}$, RCB (REGULATOR OF CHLOROPLAST BIOGENESIS) ${ }^{47}$, and NCP (NUCLEAR CONTROL OF PEP ACTIVITY) ${ }^{48}$. Both HMR and $\mathrm{RCB}$ interact directly with phyB $\mathrm{B}^{47,65}$. However, it is still unclear if these proteins can influence phyB's thermal reversion. Interestingly, HMR and PCH1 are also required for thermomorphogenesis ${ }^{50,56}$. Future investigations are needed to determine whether the modifications of phyB and the interactions between phyB and its binding partners vary among individual PBs.

While warm temperature in the light affects Pfr:Pfr indirectly by accelerating the $\mathrm{Pfr}: \mathrm{Pr}$ to $\mathrm{Pr}: \mathrm{Pr}$ reversion, shade or FR directly photoconvert Pfr:Pfr to Pfr:Pr and $\operatorname{Pr}: \operatorname{Pr}^{18}$. Therefore, it is conceivable that whereas temperature increases might not be able to disassemble certain PBs if their Pfr:Pfr is particularly stabilized, shade should be forceful enough to photoconvert phyB in all PBs. In support of this model, certain phyB mutants that block thermal reversion only failed to respond to temperature, but could still respond to shade ${ }^{18}$. Therefore, theoretically, warm temperatures could result in a PB pattern different from a shade treatment ${ }^{18}$. Our results demonstrate that warm temperatures and shade indeed elicit distinct PB dynamics. Temperature increases eliminate the localization of phyB-FPs to only selective thermosensitive PBs (Figs. 2 and 3), whereas all $\mathrm{PBs}$ disappeared in the simulated shade condition (Fig. 6a-c). It is important to point out that the $\mathrm{PB}$ responses shown in monochromatic $\mathrm{R}$ light or $\mathrm{R}$ light supplemented with FR light could be modulated in the natural light conditions wherein multiple classes photoreceptors are in action. Nonetheless, these results provide direct evidence that warm temperature and shade induce different PB dynamics. It is unclear how phyB-FP localization transitions from a few PBs to tens of small foci. Transitions between small foci and PBs also occur during the dark-to-light and light-to-dark transitions ${ }^{36,40}$, suggesting that small foci might assemble into large PBs. However, it is equally possible that PBs and small foci represent discrete sites and that phyB-FPs can redistribute between these two types of sites in a light-regulated manner. Future work will focus on investigating the mechanisms underlying the distinct PB dynamics by warm temperature and shade, as well as the consequences brought about by the distinct $\mathrm{PB}$ patterns.

\section{Methods}

Plant materials and growth conditions. The $P B G^{36}, P B C^{55}, Y H B^{53}$ and $B C Y^{55}$ lines of Arabidopsis were previously described. Seeds were surface sterilized ${ }^{65}$ and plated on half-strength Murashige and Skoog media with Gamborg's Vitamins (MSP0506, Caisson Laboratories, North Logan, UT), 0.5 mM MES (pH $5.7)$, and $0.8 \%(w / v)$ agar (A038, Caisson Laboratories, North Logan, UT). Seeds were stratified in the dark at $4{ }^{\circ} \mathrm{C}$ for 5 days before treatment of specific light and temperature in an LED chamber (Percival Scientific, Perry, IA). For temperature experiments, seedlings were grown first in $21^{\circ} \mathrm{C}$ for 2 days $(48 \mathrm{~h})$, and then either kept at $21^{\circ} \mathrm{C}$ or transferred to 12,16 , or $27^{\circ} \mathrm{C}$ for 2 additional days $(48 \mathrm{~h})$ before characterization. The light condition was maintained constant at 10 or $50 \mu \mathrm{mol} \mathrm{m}{ }^{-2} \mathrm{~s}^{-1} \mathrm{R}$ light for the entire 4 days. For the shade condition, seedlings were grown in a mixture of $10 \mu \mathrm{mol} \mathrm{m}{ }^{-2} \mathrm{~s}^{-1} \mathrm{R}$ and $10 \mu \mathrm{mol} \mathrm{m}^{-2} \mathrm{~s}^{-1}$ FR light at $21^{\circ} \mathrm{C}$ for 4 days. For dark treatment, $B C Y$ and $Y H B$ seeds were exposed to $10 \mu \mathrm{mol} \mathrm{m}{ }^{-2} \mathrm{~s}^{-1} \mathrm{FR}$ light for $3 \mathrm{~h}$ immediately after stratification to deactivate the remaining phys and induce germination 65 , and then kept in the dark at $21^{\circ} \mathrm{C}$ for $45 \mathrm{~h}$ before a treatment of an indicated temperature for 2 additional days. Fluence rates of light were measured using an Apogee PS200 spectroradiometer (Apogee Instruments Inc., Logan, UT).

Hypocotyl and cotyledon measurement. Seedlings were mounted in phosphatebuffered saline (PBS) on a slide and then scanned using an Epson Perfection V700 photo scanner. Hypocotyl length and cotyledon size were measured using the NIH ImageJ software (http://rsb.info.nih.gov/nih-image/). 
Seedling preparation and mounting. For steady-state PB analysis, seedlings were fixed following the protocol as described ${ }^{58}$ with slight modifications. Seedlings were fixed under vacuum with $1 \%$ paraformaldehyde in PBS in the same growth conditions for $10 \mathrm{~min}$. After quenching with $50 \mathrm{mM} \mathrm{NH}_{4} \mathrm{Cl}$, the fixed seedlings were permeabilized with $0.2 \%$ Triton X-100 in PBS, and nuclei were stained with $3.6 \mu \mathrm{M}$ $4^{\prime}$,6-diamidino-2-phenylindole (DAPI) in PBS for $10 \mathrm{~min}$. Seedlings were washed with PBS before mounted on a slide using Prolong ${ }^{\mathrm{TM}}$ Diamond Antifade Mountant (Thermo Fisher Scientific, Waltham, MA). The slides were left to cure overnight in the dark before being sealed with nail polish and stored at $4{ }^{\circ} \mathrm{C}$ in the dark. For all samples, we always observed both live and fixed samples, and no difference between these two conditions was observed in seedlings grown in continuous $\mathrm{R}$ light. However, we found that fixation weakened the signals of some very small PBs for the shade-treated $P B G$ samples. Therefore, we chose to characterize the shade samples using live-cell imaging. For live-cell imaging, a seedling was mounted with PBS and transported immediately to the microscope within an aluminum foilwrapped petri dish. Nuclei were imaged within 5 min of mounting.

\section{Fluorescence deconvolution microscopy and imaging analysis. Three-}

dimensional image stacks of individual nuclei from cotyledon epidermal or upper hypocotyl epidermal cells were imaged using a Zeiss Axio Observer.Z1 inverted microscope equipped with a Plan-Apochromat 100×/1.4 oil-immersion objective and an Axiocam 506 mono camera (Carl Zeiss, Jena, Germany). Fluorescence was detected by using a broad spectrum X-Cite 120LED Boost high-power LED illumination system (Excelitas Technologies Corp., Waltham, MA) and the following Zeiss filter sets: DAPI, exciter $365 \mathrm{~nm}$, emitter $445 / 50 \mathrm{~nm} / \mathrm{nm}$ (Zeiss Filter Set 49); GFP, exciter $470 / 40 \mathrm{~nm} / \mathrm{nm}$, emitter $525 / 50 \mathrm{~nm} / \mathrm{nm}$ (Zeiss Filter Set 38); CFP, exciter $436 / 25 \mathrm{~nm} / \mathrm{nm}$, emitter $480 / 40 \mathrm{~nm} / \mathrm{nm}$ (Zeiss Filter Set 47 ); YFP, exciter $500 / 25 \mathrm{~nm} / \mathrm{nm}$, emitter $535 / 40 \mathrm{~nm} / \mathrm{nm}$ (Zeiss Filter Set 46). Image stacks with a Zstep size of $0.25 \mu \mathrm{m}$ were subjected to iterative classic maximum likelihood estimation deconvolution using Huygens Essential (Scientific Volume Imaging, Netherlands) with the following parameters: 50 iterations, signal-to-noise ratio 40 , and quality threshold 0.01 . The object analyzer tool was used to threshold the image and to calculate the number and volume of the PBs. For each nucleus, the distinction between a Nuo-PB and a nonNuo-PB was manually performed. The maximum projection of the deconvolved sections was exported to TIFF (Tagged Image File Format) and then processed by Adobe Photoshop CC (Adobe, San Jose, CA).

Protein extraction and immunoblots. Total protein was extracted from Arabidopsis seedlings grown under the indicated conditions. Plant tissues were ground using a Mini-Beadbeater-24 (BioSpec Products Inc.) in three volumes $(\mathrm{mg} / \mu \mathrm{L})$ of extraction buffer containing $100 \mathrm{mM}$ Tris-Cl, $\mathrm{pH} 7.5,100 \mathrm{mM} \mathrm{NaCl}, 5 \mathrm{mM}$ EDTA, $5 \%$ sodium dodecyl sulfate (SDS), $20 \mathrm{mM}$ dithiothreitol, $40 \mathrm{mM} \beta$-mercaptoethanol, $2 \mathrm{mM}$ phenylmethylsulfonyl fluoride, $80 \mu \mathrm{M}$ MG115 (Sigma-Aldrich), $80 \mu \mathrm{M}$ MG132 (Sigma-Aldrich), 1× EDTA-free protease inhibitor cocktail (Roche), $20 \%$ glycerol, $10 \mathrm{mM} \mathrm{N}$-ethylmaleimide and $0.01 \%$ bromophenol blue and immediately boiled for $10 \mathrm{~min}$, followed by centrifugation at $16,000 \times \mathrm{g}$ for $10 \mathrm{~min}$. Proteins in the supernatant were separated by SDS-polyacrylamide gel electrophoresis and transferred to nitrocellulose membranes for immunoblotting. Primary antibodies, including monoclonal mouse anti-phyB antibodies (gift from Akira Nagatani) and polyclonal rabbit anti-RPN6 antibodies (Enzo Life Sciences, BML-PW8370-0100) were used at 1:1000 dilution. Goat anti-mouse (Bio-Rad, \#1706516) and goat antirabbit (Bio-Rad, \#1706515) secondary antibodies were used at a 1:5000 dilution. Signals were detected by chemiluminescence using a SuperSignal kit (Thermo Fisher Scientific) and films (Phenix Research Products), and the bands were quantified by using the NIH ImageJ software. The relative levels of phyB-FPs were normalized against the corresponding levels of RPN6.

Reporting summary. Further information on research design is available in the Nature Research Reporting Summary linked to this article.

\section{Data availability}

The source data underlying Figs. 1a-d, $2 \mathrm{~b}-\mathrm{f}, 3 \mathrm{~b}-\mathrm{f}, 4 \mathrm{~b}-\mathrm{e}, 5 \mathrm{~b}-\mathrm{g}$ and $\mathrm{6b}, \mathrm{c}$ are provided as a Source Data file.

Received: 5 October 2019; Accepted: 10 March 2020;

Published online: 03 April 2020

\section{References}

1. Chinnusamy, V., Zhu, J. \& Zhu, J.-K. Cold stress regulation of gene expression in plants. Trends Plant Sci. 12, 444-451 (2007).

2. Shi, Y., Ding, Y. \& Yang, S. Molecular regulation of CBF signaling in cold acclimation. Trends Plant Sci. 23, 623-637 (2018).
3. Ohama, N., Sato, H., Shinozaki, K. \& Yamaguchi-Shinozaki, K. Transcriptional regulatory network of plant heat stress response. Trends Plant Sci. 22, 53-65 (2017).

4. Wigge, P. A. Ambient temperature signalling in plants. Curr. Opin. Plant Biol. 16, 661-666 (2013)

5. Koini, M. A. et al. High temperature-mediated adaptations in plant architecture require the bHLH transcription factor PIF4. Curr. Biol. 19, 408-413 (2009).

6. Crawford, A. J., McLachlan, D. H., Hetherington, A. M. \& Franklin, K. A. High temperature exposure increases plant cooling capacity. Curr. Biol. 22, R396-R397 (2012).

7. Blázquez, M. A., Ahn, J. H. \& Weigel, D. A thermosensory pathway controlling flowering time in Arabidopsis thaliana. Nat. Genet. 33, 168-171 (2003).

8. Kumar, S. V. et al. Transcription factor PIF4 controls the thermosensory activation of flowering. Nature 484, 242-245 (2012).

9. Lau, O. S. et al. Direct control of SPEECHLESS by PIF4 in the hightemperature response of stomatal development. Curr. Biol. 28, 1273-1280.e3 (2018).

10. Gangappa, S. N., Berriri, S. \& Kumar, S. V. PIF4 coordinates thermosensory growth and immunity in Arabidopsis. Curr. Biol. 27, 243-249 (2017).

11. Huot, B. et al. Dual impact of elevated temperature on plant defence and bacterial virulence in Arabidopsis. Nat. Commun. 8, 1808 (2017).

12. Hua, J. Modulation of plant immunity by light, circadian rhythm, and temperature. Curr. Opin. Plant Biol. 16, 406-413 (2013).

13. Quint, M. et al. Molecular and genetic control of plant thermomorphogenesis. Nat. Plants 2, 15190 (2016).

14. Casal, J. J. \& Balasubramanian, S. Thermomorphogenesis. Annu. Rev. Plant Biol. 70, 321-346 (2019).

15. Nicotra, A. B. et al. Plant phenotypic plasticity in a changing climate. Trends Plant Sci. 15, 684-692 (2010).

16. Schlenker, W. \& Roberts, M. J. Nonlinear temperature effects indicate severe damages to US crop yields under climate change. Proc. Natl Acad. Sci. USA 106, 15594-15598 (2009)

17. Bailey-Serres, J., Parker, J. E., Ainsworth, E. A., Oldroyd, G. E. D. \& Schroeder, J. I. Genetic strategies for improving crop yields. Nature 575, 109-118 (2019).

18. Legris, M. et al. Phytochrome B integrates light and temperature signals in Arabidopsis. Science 354, 897-900 (2016).

19. Jung, J.-H. et al. Phytochromes function as thermosensors in Arabidopsis. Science 354, 886-889 (2016)

20. Rockwell, N. C., Su, Y. S. \& Lagarias, J. C. Phytochrome structure and signaling mechanisms. Annu. Rev. Plant Biol. 57, 837-858 (2006).

21. Burgie, E. S. \& Vierstra, R. D. Phytochromes: an atomic perspective on photoactivation and signaling. Plant Cell 26, 4568-4583 (2014).

22. Rockwell, N. C. \& Lagarias, J. C. Phytochrome evolution in 3D: deletion, duplication, and diversification. New Phytol. 225, 2283-2300 (2020).

23. Franklin, K. A. \& Whitelam, G. C. Light-quality regulation of freezing tolerance in Arabidopsis thaliana. Nat. Genet. 39, 1410-1413 (2007).

24. Ballaré, C. L. \& Pierik, R. The shade-avoidance syndrome: multiple signals and ecological consequences. Plant Cell Environ. 40, 2530-2543 (2017).

25. Legris, M., Ince, Y. Ç. \& Fankhauser, C. Molecular mechanisms underlying phytochrome-controlled morphogenesis in plants. Nat. Commun. 10, 5219 (2019).

26. Nagy, F. \& Schafer, E. Phytochromes control photomorphogenesis by differentially regulated, interacting signaling pathways in higher plants. Annu. Rev. Plant Biol. 53, 329-355 (2002).

27. Klose, C., Nagy, F. \& Schafer, E. Thermal reversion of plant phytochromes, Mol. Plant 13, 386-397 (2020).

28. Chen, M. \& Chory, J. Phytochrome signaling mechanisms and the control of plant development. Trends Cell Biol. 21, 664-671 (2011)

29. Neff, M. M. \& Van Volkenburgh, E. Light-stimulated cotyledon expansion in Arabidopsis seedlings (The role of phytochrome B.). Plant Physiol. 104, 1027-1032 (1994).

30. Reed, J. W., Nagpal, P., Poole, D. S., Furuya, M. \& Chory, J. Mutations in the gene for the red/far-red light receptor phytochrome B alter cell elongation and physiological responses throughout Arabidopsis development. Plant Cell 5, 147-157 (1993).

31. Gray, W. M., Ostin, A., Sandberg, G., Romano, C. P. \& Estelle, M. High temperature promotes auxin-mediated hypocotyl elongation in Arabidopsis. Proc. Natl Acad. Sci. USA 95, 7197-7202 (1998).

32. Paik, I., Kathare, P. K., Kim, J.-I. \& Huq, E. Expanding roles of PIFs in signal integration from multiple processes. Mol. Plant 10, 1035-1046 (2017).

33. Procko, C., Crenshaw, C. M., Ljung, K., Noel, J. P. \& Chory, J. Cotyledongenerated auxin is required for shade-induced hypocotyl growth in Brassica rapa. Plant Physiol. 165, 1285-1301 (2014).

34. Fankhauser, C. \& Chen, M. Transposing phytochrome into the nucleus. Trends Plant Sci. 13, 596-601 (2008). 
35. Van Buskirk, E. K., Decker, P. V. \& Chen, M. Photobodies in light signaling. Plant Physiol. 158, 52-60 (2012).

36. Yamaguchi, R., Nakamura, M., Mochizuki, N., Kay, S. A. \& Nagatani, A. Light-dependent translocation of a phytochrome B-GFP fusion protein to the nucleus in transgenic Arabidopsis. J. Cell Biol. 145, 437-445 (1999).

37. Kircher, S. et al. Light quality-dependent nuclear import of the plant photoreceptors phytochrome A and B. Plant Cell 11, 1445-1456 (1999).

38. Bauer, D. et al. Constitutive photomorphogenesis 1 and multiple photoreceptors control degradation of phytochrome interacting factor 3 , a transcription factor required for light signaling in Arabidopsis. Plant Cell 16, 1433-1445 (2004).

39. Van Buskirk, E. K., Reddy, A. K., Nagatani, A. \& Chen, M. Photobody localization of phytochrome B is tightly correlated with prolonged and lightdependent inhibition of hypocotyl elongation in the dark. Plant Physiol. 165, 595-607 (2014).

40. Chen, M., Schwab, R. \& Chory, J. Characterization of the requirements for localization of phytochrome B to nuclear bodies. Proc. Natl Acad. Sci. USA 100, 14493-14498 (2003).

41. Rausenberger, J. et al. An integrative model for phytochrome B mediated photomorphogenesis: from protein dynamics to physiology. PLoS ONE $\mathbf{5}$, e10721 (2010).

42. Kircher, S. et al. Nucleocytoplasmic partitioning of the plant photoreceptors phytochrome A, B, C, D, and E is regulated differentially by light and exhibits a diurnal rhythm. Plant Cell 14, 1541-1555 (2002).

43. Klose, C. et al. Systematic analysis of how phytochrome B dimerization determines its specificity. Nat. Plants 1, 15090 (2015).

44. Chen, M. et al. Arabidopsis HEMERA/pTAC12 initiates photomorphogenesis by phytochromes. Cell 141, 1230-1240 (2010).

45. Trupkin, S. A., Legris, M., Buchovsky, A. S., Rivero, M. B. T. \& Casal, J. J. Phytochrome B nuclear bodies respond to the low red to far-red ratio and to the reduced irradiance of canopy shade in Arabidopsis. Plant Physiol. 165, 1698-1708 (2014).

46. Qiu, Y. et al. Mechanism of early light signaling by the carboxy-terminal output module of Arabidopsis phytochrome B. Nat. Commun. 8, 1905 (2017).

47. Yoo, C. et al. Phytochrome activates the plastid-encoded RNA polymerase for chloroplast biogenesis via nucleus-to-plastid signaling. Nat. Commun. 10, 2629 (2019).

48. Yang, E. J. et al. NCP activates chloroplast transcription by controlling phytochrome-dependent dual nuclear and plastidial switches. Nat. Commun. 10, 2630 (2019).

49. Kaiserli, E. et al. Integration of light and photoperiodic signaling in transcriptional nuclear foci. Dev. Cell 35, 311-321 (2015).

50. Huang, H. et al. PCH1 regulates light, temperature, and circadian signaling as a structural component of phytochrome B-photobodies in Arabidopsis. Proc. Natl Acad. Sci. USA 116, 8603-8608 (2019).

51. Huang, $\mathrm{H}$. et al. $\mathrm{PCH} 1$ integrates circadian and light-signaling pathways to control photoperiod-responsive growth in Arabidopsis. Elife 5, e13292 (2016).

52. Enderle, B. et al. PCH1 and PCHL promote photomorphogenesis in plants by controlling phytochrome B dark reversion. Nat. Commun. 8, 2221 (2017).

53. Su, Y.-S. \& Lagarias, J. C. Light-independent phytochrome signaling mediated by dominant GAF domain tyrosine mutants of Arabidopsis phytochromes in transgenic plants. Plant Cell 19, 2124-2139 (2007).

54. Matsushita, T., Mochizuki, N. \& Nagatani, A. Dimers of the N-terminal domain of phytochrome B are functional in the nucleus. Nature 424, 571-574 (2003).

55. Chen, M., Tao, Y., Lim, J., Shaw, A. \& Chory, J. Regulation of phytochrome B nuclear localization through light-dependent unmasking of nuclearlocalization signals. Curr. Biol. 15, 637-642 (2005).

56. Qiu, Y., Li, M., Kim, R. J.-A., Moore, C. M. \& Chen, M. Daytime temperature is sensed by phytochrome B in Arabidopsis through a transcriptional activator HEMERA. Nat. Commun. 10, 140 (2019).

57. Blum, D. E., Neff, M. M. \& Van Volkenburgh, E. Light-stimulated cotyledon expansion in the blu3 and hy4 mutants of Arabidopsis thaliana. Plant Physiol. 105, 1433-1436 (1994).

58. Yoo, C. Y., Williams, D. \& Chen, M. Quantitative analysis of photobodies. Methods Mol. Biol. 2026, 135-141 (2019).

59. Grima, R. et al. Insight into nuclear body formation of phytochromes through stochastic modelling and experiment. Phys. Biol. 15, 056003 (2018).

60. Sharrock, R. A. \& Clack, T. Heterodimerization of type II phytochromes in Arabidopsis. Proc. Natl Acad. Sci. USA 101, 11500-11505 (2004).

61. Liu, P. \& Sharrock, R. A. Directed dimerization: an in vivo expression system for functional studies of type II phytochromes. Plant J. 75, 915-926 (2013).

62. Fischer, A. J. \& Lagarias, J. C. Harnessing phytochrome's glowing potential Proc. Natl Acad. Sci. USA 101, 17334-17339 (2004).
63. Fischer, A. J. et al. Multiple roles of a conserved GAF domain tyrosine residue in cyanobacterial and plant phytochromes. Biochemistry 44, 15203-15215 (2005).

64. Hu, W., Su, Y. S. \& Lagarias, J. C. A light-independent allele of phytochrome B faithfully recapitulates photomorphogenic transcriptional networks. Mol. Plant 2, 166-182 (2009).

65. Galvao, R. M. et al. Photoactivated phytochromes interact with HEMERA and promote its accumulation to establish photomorphogenesis in Arabidopsis. Genes Dev. 26, 1851-1863 (2012).

66. Feng, C. M., Qiu, Y., Van Buskirk, E. K., Yang, E. J. \& Chen, M. Lightregulated gene repositioning in Arabidopsis. Nat. Commun. 5, 3027 (2014).

67. $\mathrm{Ni}, \mathrm{W}$. et al. A mutually assured destruction mechanism attenuates light signaling in Arabidopsis. Science 344, 1160-1164 (2014).

68. Sun, N. et al. Arabidopsis SAURs are critical for differential light regulation of the development of various organs. Proc. Natl Acad. Sci. USA 113, 6071-6076 (2016).

69. Johansson, H. et al. Arabidopsis cell expansion is controlled by a photothermal switch. Nat. Commun. 5, 4848 (2014).

70. Medzihradszky, M. et al. Phosphorylation of phytochrome B inhibits lightinduced signaling via accelerated dark reversion in Arabidopsis. Plant Cell 25, 535-544 (2013).

71. Nito, K., Wong, C. C. L., Yates, J. R. 3rd \& Chory, J. Tyrosine phosphorylation regulates the activity of phytochrome photoreceptors. Cell Rep. 3, 1970-1979 (2013).

72. Viczián, A. et al. Differential phosphorylation of the N-terminal extension regulates phytochrome B signaling. New Phytol. 42, 606-617 (2019).

73. Sweere, U. et al. Interaction of the response regulator ARR4 with phytochrome B in modulating red light signaling. Science 294, 1108-1111 (2001).

\section{Acknowledgements}

We thank Ruth Jean Ae Kim, Chan Yul Yoo, Soeun Han, and Isaac Diaz for their critical comments and suggestions regarding the manuscript. This work was supported by Grant 2R01GM087388 from the National Institute of General Medical Sciences to M.C.

\section{Author contributions}

J.H., K.K., and M.C. conceived the original research plan; J.H. and K.K. characterized the PB morphology and thermomorphogenic responses; Y.Q. and K.K. performed the immunoblot experiments; J.H., K.K., Y.Q., and M.C. analyzed the data; M.C., J.H., and K.K. wrote the article with contributions from all authors.

\section{Competing interests}

The authors declare no competing interests.

\section{Additional information}

Supplementary information is available for this paper at https://doi.org/10.1038/s41467020-15526-z.

Correspondence and requests for materials should be addressed to M.C.

Peer review information Nature Communications thanks Christian Fankhauser, Jeong-Il Kim and the other, anonymous, reviewer(s) for their contribution to the peer review of this work. Peer reviewer reports are available.

Reprints and permission information is available at http://www.nature.com/reprints

Publisher's note Springer Nature remains neutral with regard to jurisdictional claims in published maps and institutional affiliations.

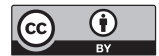

Open Access This article is licensed under a Creative Commons Attribution 4.0 International License, which permits use, sharing, adaptation, distribution and reproduction in any medium or format, as long as you give appropriate credit to the original author(s) and the source, provide a link to the Creative Commons license, and indicate if changes were made. The images or other third party material in this article are included in the article's Creative Commons license, unless indicated otherwise in a credit line to the material. If material is not included in the article's Creative Commons license and your intended use is not permitted by statutory regulation or exceeds the permitted use, you will need to obtain permission directly from the copyright holder. To view a copy of this license, visit http://creativecommons.org/ licenses/by/4.0/.

(C) The Author(s) 2020 